Aus der Abteilung Klinische Neurophysiologie

(Prof. Dr. med. W. Paulus)

im Zentrum Neurologische Medizin

der Medizinischen Fakultät der Universität Göttingen

\title{
Neuroplastische Effekte repetitiver anodaler transkranieller Gleichstromstimulation des motorischen Kortex
}

\author{
INAUGURAL-DISSERTATION \\ zur Erlangung des Doktorgrades \\ der Medizinischen Fakultät \\ der Georg-August-Universität zu Göttingen
}

vorgelegt von

Silvia Hessenthaler

aus Kassel

Göttingen 2012 
Dekan:

1. Berichterstatter:

2. Berichterstatter:
Prof. Dr. med. M. P. Schön

\author{
Prof. Dr. med. M. Nitsche
}

Prof. Dr. rer. nat. Keller

Tag der mündlichen Prüfung: 28.01.2013 
Die Ergebnisse dieser Arbeit wurden veröffentlicht in:

Monte-Silva K, Kuo MF, Hessenthaler S, Fresnoza S, Liebetanz D, Paulus W, Nitsche MA (2012): Induction of late LTP-like plasticity in the human motor cortex by repeated non-invasive brain stimulation. Brain Stimulation; im Druck 


\section{Inhaltsverzeichnis}

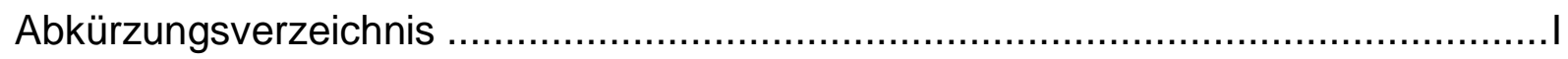

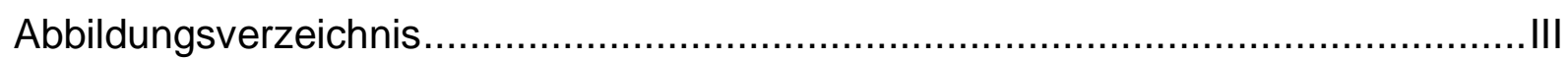

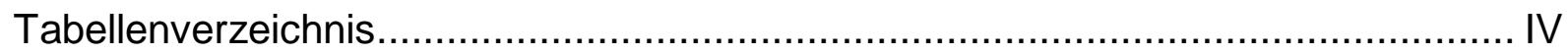

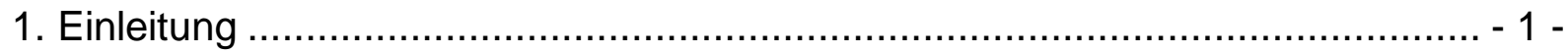

1.1 Die transkranielle Gleichstromstimulation (tDCS) .................................... - 2 -

1.1.1 Entdeckung und Funktionsweise ..................................................... - 2 -

1.1.2 Sicherheitsaspekte der tDCS ........................................................... 5 -

1.1.3 Überblick bisheriger humanexperimenteller tDCS-Studien ..................... - 6 -

1.1.3.1 Klinische Studien .................................................................... 7 -

1.1.3.2 Studien zur Lern- und Gedächtnisformierung ................................ - 9 -

1.1.3.3 Bisherige Studien zu repetitiver tDCS ........................................ - 12 -

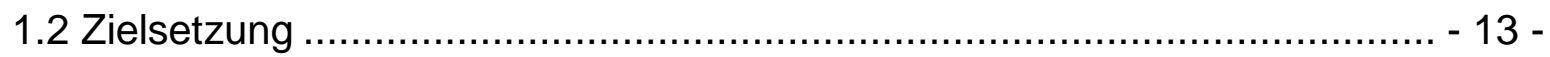

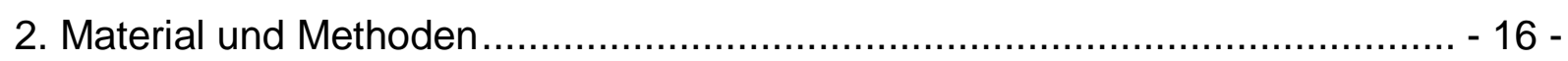



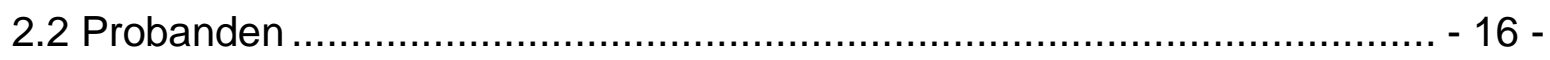

2.3 TMS über dem motorischen Kortex.................................................... - 17 -

2.4 tDCS über dem motorischen Kortex....................................................... - 18 -



2.6 Versuchsdurchführungen ..................................................................... 19 -



2.6.2 Experiment 2 …........................................................................ 21 -

2.7 Datenauswertung und Statistik .............................................................. 22 -

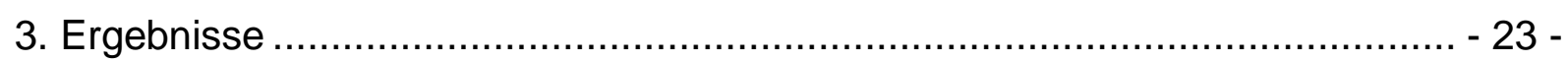

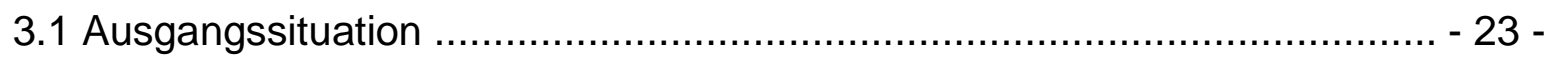

3.2 Ergebnisse des Experiments 1 ........................................................... 23 -

3.3 Ergebnisse des Experiments 2 ............................................................ 28 -

3.4 Zusammenfassung des Ergebnisteils .................................................. - 30 -

3.5 Verträglichkeit der tDCS .................................................................. 31 -

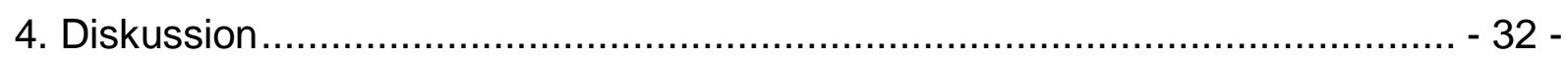

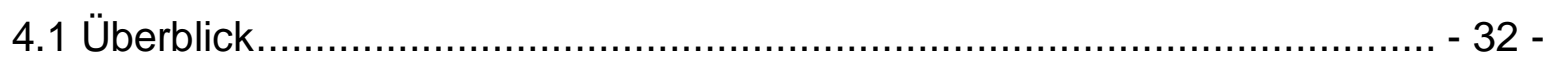

4.2 Kontinuierliche 13-minütige anodale tDCS ............................................. - 33 -

4.3 Repetitive anodale tDCS mit kurzen Pausen ............................................. - 34 -

4.4 Repetitive anodale tDCS mit langen Pausen ......................................... - 37 - 
4.5 Kontinuierliche 26-minütige anodale tDCS ......................................... - 40 -

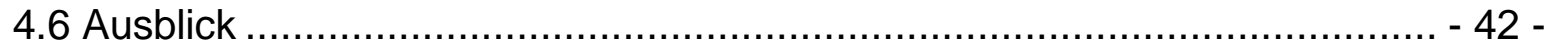

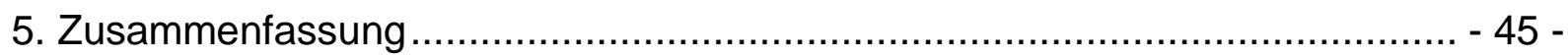

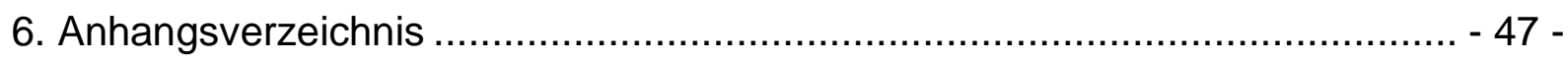

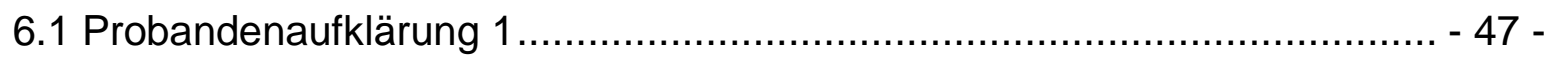

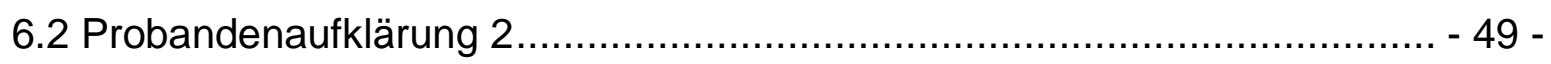

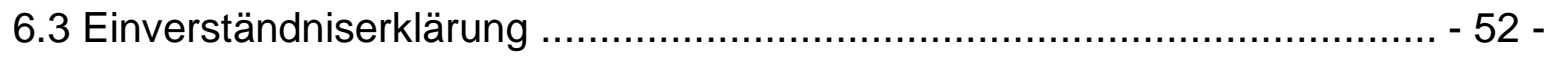

6.4 Weitere Abbildungen und Tabellen .................................................. - 53 -

7. Literaturverzeichnis ............................................................................. 61 - 


\section{Abkürzungsverzeichnis}

ADM Musculus abductor digiti minimi

AMPA a-amino-3-hydroxy-5-methyl-4-isoxazol-Propionsäure

ANOVA Varianzanalyse (Analysis of variance)

Baseline MEP-Ausgangsmesswert

BCM Bienenstock-Cooper-Munro-Regel

BDNF vom Gehirn stammender neurotropher Faktor= Wachstumsfaktor (brain-derived neurotrophic factor)

bzw. beziehungsweise

ca. circa

$\mathrm{Ca}^{2+} \quad$ Kalzium zweifach ionisiert

CaMK Kalzium-Calmodulin-Kinase

CAMP zyklisches Adenosinmonophosphat

CREB CAMP-abhängiger Transkriptionsfaktor (cAMP response elementbinding protein)

d.f. $\quad$ Freiheitsgrade (degrees of freedom)

d.h. das heißt

DLPFC dorsolateraler präfrontaler Kortex

DNA Desoxyribonukleinsäure (deoxyribonucleic acid)

EEG Elektroenzephalogramm

FLU Flunarizin

h Stunde(n)

$\mathrm{Hz} \quad$ Hertz

LTD Langzeitdepression (long-term depression)

LTP Langzeitpotenzierung (long-term potentiation)

e-LTP Frühphase der Langzeitpotenzierung (early-phase of long-term potentiation)

I-LTP Spätphase der Langzeitpotenzierung (late-phase of long-term potentiation)

$\mathrm{mA} \quad$ Milliampere

$\mu \mathrm{A} \quad$ Mikroampere

MAPK Mitogen-aktivierte Proteinkinase (mitogen-activated protein kinase) MEP motorisch evoziertes Potential 


$\begin{array}{ll}\text { mg } & \text { Milligramm } \\ \text { min } & \text { Minute(n) } \\ \text { ms } & \text { Millisekunde(n) } \\ \text { mV } & \text { Millivolt } \\ \mathrm{n} & \text { Stichprobenumfang } \\ \text { na } & \text { Nachmittag des Folgetages der letzten anodalen Gleichstromstimulation } \\ & \text { (next afternoon) } \\ \text { NaCl } & \text { Natriumchlorid } \\ \text { ne } & \text { Abend des Folgetages der letzten anodalen Gleichstromstimulation } \\ & \text { (next evening) } \\ \text { nm } & \text { Morgen des Folgetages der letzten anodalen Gleichstromstimulation } \\ & \text { (next morning) } \\ \text { NMDA } & \text { N-Methyl-D-Aspartat } \\ \text { Nr. } & \text { Nummer } \\ \text { o.g. } & \text { oben genannt } \\ \text { PAS } & \text { gepaarte assoziative Stimulation } \\ \text { p.o. } & \text { per os } \\ \text { RNA } & \text { Ribonukleinsäure (ribonucleic acid) } \\ \text { (r)TMS } & \text { (repetitive) transkranielle Magnetstimulation } \\ \text { SD } & \text { Standardabweichung } \\ \text { se } & \text { Abend des Stimulationstages (same evening) } \\ \text { SEM } & \text { Standardfehler } \\ \text { SEP } & \text { somatosensibel evoziertes Potential } \\ \text { S.O. } & \text { siehe oben } \\ \text { TBS } & \text { Theta-Burst-Stimulation } \\ \text { tDCS } & \text { transkranielle Gleichstromstimulation (transcranial direct current } \\ & \text { stimulation) } \\ \text { TNF a } & \text { Tumornekrosefaktor } \alpha \\ \text { UK } & \text { Vereinigtes Königreich Großbritannien und Nordirland (United Kingdom) } \\ \text { ZNS } & \text { zentrales Nervensystem } \\ & \end{array}$




\section{Abbildungsverzeichnis}

Abbildung 1: Kontinuierliche anodale tDCS (13/26 min) ............................... - 25 -

Abbildung 2: Repetitive anodale tDCS mit kurzen Pausen ............................. - 26 -

Abbildung 3: Repetitive anodale tDCS mit langen Pausen ........................... - 27 -

Abbildung 4: Kontinuierliche anodale tDCS (26 min)+/- Medikation .................... - 29 -

Abbildung 5: Versuchsablauf der Experimente 1 und 2 .............................. 53 - 


\section{Tabellenverzeichnis}

Tabelle 1: Baseline MEP-Amplitudenhöhe \& TMS-Intensität Experiment $1 \ldots \ldots \ldots$ - 24 -



Tabelle 3: Ergebnisse der ANOVA des Experiments 2 ................................. - 29 -

Tabelle 4: Erhobene Messwerte der Experimente 1 und 2 vor und nach tDCS ... - 55 -

Tabelle 5: $p$-Werte der Fisher-LSD-Test-Berechnung für das Experiment 1 ....... - 58 -

Tabelle 6: p-Werte der Fisher-LSD-Test-Berechnung für das Experiment 2 ....... - 60 - 


\section{Einleitung}

Das menschliche Gehirn und seine Funktionsweise geben auch im frühen 21. Jahrhundert viele Rätsel auf und bleiben ein spannendes Feld für die Neurowissenschaften. Noch immer hat man keine Erklärung für viele Vorgänge innerhalb neuronaler Netzwerke, und viele neurologische Krankheiten warten auf ein besseres Verständnis der Pathophysiologie und neue Therapiemöglichkeiten.

Genauso wichtig wie die Aufklärung der Prozesse zwischen Hirnregionen, neuronalen Netzwerken und einzelnen Synapsen ist die Anwendung von möglichst leicht durchführbaren, sicheren und nebenwirkungsarmen Verfahren am Patienten.

Nachdem die transkranielle Gleichstromstimulation für einen langen Zeitraum aus dem Blickfeld der Wissenschaft verschwunden war, ist sie mit ihrer "Renaissance“ vor ca. 15 Jahren wieder in den Fokus internationaler neurologischer Forschungsgruppen gerückt. Seitdem ist sie Bestandteil von neurophysiologischen Studien des motorischen Kortex, des visuellen Kortex, von Verhaltensstudien und findet Anwendung in Studien verschiedener klinischer Krankheitsbilder wie zum Beispiel der Depression, der Migräne, des apoplektischen Insults und des Morbus Parkinson. Sie hat zeigen können, dass ein geringer Gleichstrom im Tier und im Menschen dazu in der Lage ist, nachhaltige neuronale Plastizitätsveränderungen zu bewirken, und kann einige erfolgreiche Ergebnisse in klinischen Studien aufweisen. Die bislang erzielten Resultate geben Anlass genug, sich weiterhin intensiv mit dieser Methode der elektrischen Gehirnstimulation auseinanderzusetzen, ihre Funktionsweise noch besser zu verstehen, die Frage nach der Sicherheit dieser Methode genauer zu beleuchten und sie bei ausgewiesenem Nutzen zur Behandlung von Patienten verschiedener medizinischer Fachgebiete einzusetzen.

Um zur Weiterentwicklung der Methode und dem Verständnis ihrer zugrunde liegenden neuronalen Mechanismen beizutragen, habe ich mich mit der Frage der Effektivität repetitiver anodaler transkranieller Gleichstromstimulation in dieser Arbeit auseinandergesetzt. Da diese Stimulationsform bislang in nur wenigen Studien untersucht wurde, möchte ich durch die Anwendung verschiedener Stimulationsbedingungen ihre Wirkungsweise und Effekte auf den motorischen Kortex gesunder Probanden genauer untersuchen. 
Zunächst werde ich am Anfang meiner Arbeit die zurzeit vorliegenden Studienergebnisse zur transkraniellen Gleichstromstimulation beschreiben und den aktuellen Stand der Forschung darlegen.

Dabei werde ich die bisher bekannten Wirkmechanismen der tDCS beleuchten, Daten zur Sicherheitslage der Methode präsentieren und einen Überblick über die verschiedenen Anwendungsgebiete der transkraniellen Gleichstromstimulation liefern. Meine Schwerpunkte setze ich zum einen auf den klinischen Einsatz der Methode, um den Nutzen der Gleichstromstimulationsforschung hervorzuheben, und zum anderen auf die Studien zur Lern- und Gedächtnisformierung, da die repetitive tDCS - wie noch später in der Diskussion zu sehen sein wird - dort interessante neue Ansatzpunkte erkennen lässt.

Zusätzlich werde ich die Ergebnisse der durchgeführten repetitiven tDCS-Studien vorstellen und am Ende der Einleitung die Zielsetzung meiner Arbeit formulieren.

\subsection{Die transkranielle Gleichstromstimulation (tDCS)}

\subsubsection{Entdeckung und Funktionsweise}

Die Möglichkeit, eine elektrische Stimulation des Gehirns transkraniell durchzuführen, ist seit den 1960er Jahren bekannt. Zunächst wurden die Auswirkungen eines schwachen Gleichstroms auf die neuronale Aktivität im Tierversuch an Katzen und Ratten untersucht. Die Elektroden wurden dabei beim anästhesierten Tier direkt auf der Kortexoberfläche angebracht. Mehrere Forschungsgruppen konnten nachweisen, dass ein schwacher Stromfluss die neuronale Aktivität und Entladungsrate verändert, wobei eine anodale Stimulation zu einer Steigerung der Spontanaktivität führte und eine kathodale Stimulation eine Reduktion der neuronalen Aktivität zur Folge hatte (Bindman et al. 1964, Creutzfeldt et al. 1962, Purpura und Mc Murtry 1965). Als ursächlicher Mechanismus ist die Verschiebung des neuronalen Ruhemembranpotentials im Sinne einer unterschwelligen De- oder Hyperpolarisation zu benennen (Purpura und Mc Murtry 1965). Diese frühen Experimente zur tDCS lieferten außerdem die wichtige Erkenntnis, dass die Effektivität der Stimulation von der Stromflussrichtung, der Orientierung der dadurch stimulierten Neuronen und der Stimulationsintensität abhängig ist. Neben den oben beschriebenen Nettoeffekten der tDCS präsentierten sich für anders strukturierte und lokalisierte Neuronenpopulationen entgegengesetzte Effekte. Im Motorkortex der Katze 
resultierte eine anodale Stimulation in den tiefer gelegenen Neuronenpopulationen in einer verminderten neuronalen Aktivität und in entgegengesetzter Weise führte eine kathodale Stimulation zu einer Aktivitätssteigerung der Neurone (Creutzfeldt et al. 1962). In der Studie von Purpura und Mc Murtry 1965 führte die anodale Stimulation von oberflächlich gelegenen Pyramidenzellen - im Gegensatz zu den tiefer gelegenen Pyramidenzellen - zu einer Hyperpolarisation und eine schwache kathodale Gleichstromstimulation zu einer Depolarisation. Darüber hinaus zeigte sich, dass nicht-pyramidale Neurone schon auf geringe Stromintensitäten mit Veränderungen in ihrem Aktivitätslevel reagierten, wohingegen für Pyramidenzellen höhere Intensitäten zur Veränderung des Erregungszustandes nötig waren (Purpura und Mc Murtry 1965).

Nach zahlreichen tierexperimentellen Studien begann man schließlich damit, die schwache Gleichstromstimulation auch am Menschen zu untersuchen. Man versuchte, die tDCS in der Behandlung von psychiatrischen Erkrankungen wie der Depression und der Manie einzusetzen (Carney 1969; Costain et al. 1964; Lolas 1977). Trotz einiger vielversprechender Ergebnisse wurden keine größeren und systematischen Studien angeschlossen, sodass die tDCS für einige Zeit in Vergessenheit geriet und erst in den letzten zwei Jahrzehnten wieder für Aufmerksamkeit in den Neurowissenschaften sorgt. Konträr zu den Applikationsformen im Tierversuch wird der Gleichstrom humanexperimentell über an der Kopfhaut befestigte Elektroden mit einer Größe zwischen $25-35 \mathrm{~cm}^{2}$ geleitet und stellt damit eine nicht-invasive Stimulationsform dar. Zur Vermeidung eines zu starken Juckens oder Kribbelns unter den Elektroden werden mit $0,9 \% \mathrm{NaCl}$ befeuchtete Schwammelektroden verwendet. Die Stromstärke in aktuellen Studien variiert zwischen 1-2 mA bei einer Stromdichte zwischen 0,029-0,08 mA/ $\mathrm{cm}^{2}$ (Nitsche et al. 2008). Von dem transkraniell applizierten Stromfluss erreichen ca. $50 \%$ das menschliche Gehirn (Rush und Driscoll 1968). Der Strom fließt von dem negativen Pol (Kathode) zum positiven Pol (Anode). Dabei wird unter den Elektroden ein elektrisches Feld generiert, welches relativ homogen ist und dessen Feldstärke exponentiell mit der Entfernung zu diesen abnimmt (Miranda et al. 2006; Rush und Driscoll 1968).

tDCS bewirkt in Analogie zu den Ergebnissen der Tierversuche innerhalb des menschlichen Gehirns eine unterschwellige, tonische Verschiebung des neuronalen Ruhemembranpotentials, die in einer De- oder Hyperpolarisation mündet (Nitsche et 
al. 2003a). Zudem reagiert auch der Motorkortex des Menschen mit einer kortikalen Erregbarkeitssteigerung durch anodale Gleichstromstimulation und einer Erregbarkeitsverminderung durch kathodale tDCS (Nitsche und Paulus 2000, 2001; Nitsche et al. 2003b). Bisher konnte tDCS nicht nur während der Stimulation Veränderungen der neuronalen Aktivität, sondern auch über die Stimulation hinaus andauernde Nacheffekte erzeugen. Dabei führte kontinuierliche 13-minütige anodale Gleichstromstimulation zu einer Erregbarkeitssteigerung des motorischen Kortex bis zur 90. Minute nach Stimulationsende und kontinuierliche 9-minütige kathodale Gleichstromstimulation zu einer Erregbarkeitsverminderung für eine ebenso lange Zeit (Nitsche und Paulus 2000, 2001). Auch in den frühen Tierversuchen wurde bereits die Fähigkeit der tDCS zur Erzeugung langanhaltender Nacheffekte von mehreren Stunden demonstriert (Bindman et al. 1964). Diese langanhaltenden Effekte scheinen entsprechend tierexperimentellen Befunden nicht ausschließlich auf Veränderungen des neuronalen Ruhemembranpotentials, sondern auch auf neuer Proteinsynthese zu beruhen (Gartside 1968). Zusätzlich lieferten weitere Studien die Ergebnisse, dass mehrminütige kontinuierliche anodale tDCS zu einer Veränderung der Noradrenalin-getriggerten cAMP-Konzentration und intrazellulärer Kalziumakkumulation führt (Hattori et al. 1990; Islam et al. 1995). Darüber hinaus sind die neuronalen Erregbarkeitsveränderungen durch tDCS beim Menschen von der Effektivität der NMDA-Rezeptoren abhängig, was mit Hilfe einiger pharmakologischer Studien belegt werden konnte (Liebetanz et al. 2002; Nitsche et al. 2003a, 2004a). Aufgrund der ähnlichen Wirkungsweise könnten die bis zu einer Stunde anhaltenden Nacheffekte erregbarkeitssteigernder kortikaler Aktivität anodaler tDCS ein Ausdruck gesteigerter synaptischer Übertragungsraten, d.h. der Langzeitpotenzierung (LTP), sein und ebenso die durch kathodale tDCS induzierte Abnahme kortikaler Erregbarkeit ein Ausdruck einer verminderten synaptischen Effizienz, also der Langzeitdepression (LTD), sein. Daher werden diese durch tDCS ausgelösten neuroplastischen Veränderungen üblicherweise als LTP-/LTD-ähnliche Plastizität bezeichnet (Malenka und Bear 2004; Nitsche und Paulus 2000, 2001).

Zusammenfassend wirkt tDCS als neuromodulatorisches Stimulationsverfahren über eine Verschiebung des neuronalen Ruhemembranpotentials und über die Veränderung der Effektivität spannungsabhängiger lonenkanäle. Die Modifikation der NMDA-Rezeptoreffektivität führt zu einer veränderten Stärke glutamaterger synaptischer Verbindungen und darauf folgender exzitatorischer Aktionspotentiale. 
Die bislang bekannten, abhängigen Variablen für die Größe, Richtung und den zeitlichen Verlauf einer applizierten Gleichstromstimulation beim Menschen sind die Polarität der Stimulation (anodal oder kathodal), die Stromflussrichtung in Abhängigkeit von der Positionierung der Elektroden, die verwendete Stromstärke, die Stromdichte und die Dauer der Stimulation (Nitsche et al. 2008; Stagg und Nitsche 2011).

\subsubsection{Sicherheitsaspekte der tDCS}

Aktuell gibt es keine systematische Analyse für die Grenzen einer sicheren Gleichstromstimulation am Menschen. Dennoch gibt es einige Studien, die sich mit den Risiken und Nebenwirkungen der am häufigsten eingesetzten Stimulationsparameter beschäftigt haben und zu folgenden Ergebnissen kommen: Als sicher zu bewerten gelten Stimulationsbedingungen mit einer Stromstärke von bis zu $2 \mathrm{~mA}$, einer Stimulationsdauer bis zu 20 Minuten, einer Elektrodengröße zwischen $25-35 \mathrm{~cm}^{2}$ und einer Stromdichte von bis zu $0,029 \mathrm{~mA} / \mathrm{cm}^{2}$ (Nitsche et al. 2008). Diese führten weder zu Hitzeeffekten mit Gewebsschädigung unter den Elektroden (Nitsche und Paulus 2000), noch zu einer Erhöhung der Konzentration der neuronenspezifischen Enolase (Nitsche und Paulus 2001; Nitsche et al. 2003b) einem sensitiven Marker für neuronale Schädigungen (Steinhoff et al. 1999). Unter diesen Voraussetzungen waren ebenso keine Potentialveränderungen im EEG oder Wesensveränderungen zu beobachten (lyer et al. 2005; Nitsche et al. 2003c). Weiterhin stellte eine MRT-Studie fest, dass tDCS keine strukturellen Gewebeschäden des Hirnparenchyms, ein Hirnödem oder Veränderungen in der Blut-Hirn Schranke nach sich zieht (Nitsche et al. 2004b).

Ferner konnte eine Sicherheitsstudie zu kathodaler tDCS an Ratten nachweisen, dass bei einer Stromintensität zwischen 142,9-287 A/m ${ }^{2}$ bis zu einem Schwellenwert der elektrischen Ladungsdichte von $52400 \mathrm{C} / \mathrm{m}^{2}$, die um ein Vielfaches über dem Niveau der humanexperimentell angewandten Strom- $\left(0,3-0,8 \mathrm{~A} / \mathrm{m}^{2}\right)$ und Ladungsdichten lagen $\left(171-480 \mathrm{C} / \mathrm{m}^{2}\right)$, keine morphologischen Hirnschäden tierexperimentell auftraten. Darüber hinaus führte eine Stromdichte von bis zu 28,6 $\mathrm{A} / \mathrm{m}^{2}$ über eine Dauer von 270 Minuten zu keiner pathologischen Gewebsschädigung im Rattenmodell (Liebetanz et al. 2009). 
Weltweit wurden diese tDCS-Formen an ca. 2000-3000 Probanden ohne ernsthafte Komplikationen oder unerwünschte Wirkungen angewandt. Es traten dabei lediglich leichte Nebenwirkungen wie Kribbeln oder Jucken unter den Elektroden, leichte Müdigkeit, milde Kopfschmerzen und selten Übelkeit auf (Poreisz et al. 2007). Nichtsdestotrotz sollte man einige Hinweise beachten, um die Gesundheit der Probanden und Patienten nicht zu gefährden:

In einer Studie von 2008 wurden bei einigen Patienten Hautläsionen unter den Elektroden festgestellt, weshalb vor der Anwendung dieser Methode die Patienten nach dermatologischen Erkrankungen befragt und die Kopfhaut vor und nach der Stimulation untersucht werden sollte (Nitsche et al. 2008).

Häufig tritt nach der Applikation von tDCS eine leichte Rötung unter den Elektroden auf, welche jedoch auf vasodilatatorische Effekte zurückzuführen ist und kein Anzeichen eines Hautschadens bedeutet (Durand et al. 2002). Ferner muss die Elektrodenposition so gewählt werden, dass tDCS nicht über Foramina, Fissuren, offenen Fontanellen und Schädeldefekten appliziert wird, da dies bei Fokussierung des Stromflusses zu Parenchymschäden führen könnte (Rush und Driscoll 1968). Eine Stimulation des Hirnstamms muss unbedingt vermieden werden und die Auswahl der Elektrodenpositionen sollte sorgfältig getroffen werden (Lippold und Redfearn 1964). Abschließend müssen vor der Teilnahme an einer tDCS-Studie die Patienten sorgfältig auf Ausschlusskriterien wie Schwangerschaft, ernsthafte neurologische (z.B. Epilepsie) und psychiatrische Erkrankungen, Suchterkrankungen, Metallimplantate innerhalb des Kopfes und schwerwiegende internistische Erkrankungen befragt werden (Nitsche et al. 2008).

Sofern diese Aspekte zur Sicherheit der tDCS berücksichtigt werden und solche Stimulationsformen angewandt werden, die momentan als sicher gelten, ist die transkranielle Gleichstromstimulation als risikoarm zu bewerten.

\subsection{3 Überblick bisheriger humanexperimenteller tDCS-Studien}

Der größte Anteil der bisher durchgeführten Studien am Menschen untersuchte die Effekte transkranieller Gleichstromstimulation am motorischen Kortex. Diese Ergebnisse wurden bereits im vorherigen Teil dargelegt. Darüber hinaus beschäftigten sich viele Forschungsgruppen auch mit der Beeinflussung anderer kortikaler Areale wie beispielsweise des visuellen und des somatosensorischen 
Kortex. Innerhalb des menschlichen visuellen Kortex konnte durch mehrminütige transkranielle Gleichstromstimulation eine Veränderung der Kontrastwahrnehmung, des Schwellenwertes zur Auslösung von Phosphenen und der beta- und gammaOszillationen erzielt werden (Antal et al. 2001, 2003, 2004a).

Eine 9-minütige kathodale transkranielle Gleichstromstimulation über dem somatosensorischen Kortex lieferte eine Verminderung der N20-Komponente des SEPs des N. medianus, die bis zu einer Stunde nach Stimulationsende nachweisbar war (Dieckhöfer et al. 2006). Rogalewski et al. zeigten 2004, dass eine 7-minütige kathodale tDCS über dem somatosensorischen Kortex zu einer verringerten taktilen Diskriminationsfähigkeit führte - verglichen mit einer Plazebostimulation oder einer anodalen Stimulationssituation.

\subsubsection{Klinische Studien}

Auch in der klinischen Anwendung kann die transkranielle Gleichstromstimulation erfolgreiche Ergebnisse bei den Krankheiten Depression, Epilepsie, Schlaganfall, Schmerzwahrnehmung, Migräne, Morbus Parkinson, Alzheimer und Tinnitus aufweisen, wie Pilotstudien zeigen (Antal et al. 2011; Boggio et al. 2009; Fregni et al. 2006a, b, c, d, e; Hummel et al. 2005; Nitsche et al. 2009; Vanneste et al. 2010). Diese Erkrankungen teilen auf kortikaler Ebene die Gemeinsamkeit, dass in bestimmten Gehirnarealen eine pathologische neuronale Plastizität und Hypo- oder Hyperexzitabilität auftritt (z.B. eine gesteigerte Erregbarkeit der Hirnrinde bei Parkinson-, Epilepsie- oder Migränepatienten (Nitsche et al. 2002)), an welcher die transkranielle Gleichstromstimulation durch entgegengesetzte Modulation der kortikalen Erregbarkeit ansetzt. Die bisherigen Resultate der klinischen Studien werde ich im Folgenden detailliert darlegen.

In der ersten randomisierten, doppelblinden, plazebokontrollierten Studie an 10 Patienten mit neu diagnostizierter Major Depression ohne bisherige antidepressive Medikation konnten Fregni et al. (2006b) zeigen, dass eine 20-minütige anodale tDCS von $1 \mathrm{~mA}$ über dem dorsolateralen präfrontalen Kortex an fünf aufeinanderfolgenden Tagen zu einer signifikanten Reduktion der Depressionssymptome anhand der Hamilton Depression Rating Scale (HAMD) und des Beck Depression Inventory Scores (BDI) führte. Darüber hinaus zeigte eine weitere Studie, die die Effektivität von tDCS gegenüber antidepressiver Medikation untersuchte, eine gleichartige Wirksamkeit beider Methoden (Rigonatti et al. 2008). 
Die Reduktion der depressiven Symptome war sowohl für eine 20-minütige anodale tDCS über dem linken dorsolateralen präfrontalen Kortex mit einer Stromintensität von $0,057 \mathrm{~mA} / \mathrm{cm}^{2}$ über zehn Tage als auch für die tägliche Einnahme von $20 \mathrm{mg}$ Fluoxetin über sechs Wochen signifikant. Allerdings unterschieden sich die Therapiestrategien im Auftreten ihres Maximaleffektes, der bei anodaler tDCS sofort nach Stimulation zu verzeichnen war und bis zu weiteren vier Wochen konstant blieb, wohingegen sich der Höhepunkt der Wirksamkeit für Fluoxetin erst nach sechs Wochen manifestierte. Die Autoren der Studie folgerten daraus, dass tDCS bei der Behandlung von Depressionen möglicherweise zur Überbrückung bis zur maximalen Wirksamkeit eines Antidepressivums eine wichtige Therapieoptimierung sein könnte. Ferner präsentieren sich folgende Ergebnisse innerhalb der Epilepsieforschung: Für das Rampen-Stimulations-Modell der fokalen Epilepsie konnte im Tierversuch an Ratten demonstriert werden, dass eine 60 -minütige kathodale tDCS von $100 \mu \mathrm{A}$ oder eine 30-minütige kathodale tDCS von $200 \mu \mathrm{A}$ die antikonvulsive Schwelle bis zu 120 Minuten nach Stimulationsende erhöhen kann (Liebetanz et al. 2006). Innerhalb einer klinischen Studie an Patienten mit pharmakoresistenter Epilepsie aufgrund von Malformationen des Gehirns erzeugte eine 20-minütige kathodale tDCS von $1 \mathrm{~mA}$ über dem epileptischen Fokus eine signifikante Reduktion der epileptoformen Entladungsfrequenz und Feuerrate (Fregni et al. 2006c; Nitsche und Paulus 2009). Weiterhin widmet sich die tDCS-Forschung dem Anwendungsbereich des apoplektischen Insults. 2010 zeigten Kim SJ et al. im Tierversuch am Rattenmodell mit zerebrovaskulärem Insult, dass eine 30-minütige anodale tDCS von 0,1 mA über zwei Wochen zu einer verbesserten motorischen Funktion innerhalb des Garcia'sTest und Foot-fault-Test, sowie histologisch zu einer geringeren Axonzerstörung führte. Darüber hinaus realisierten Hummel et al. 2005 eine doppelblinde, plazebokontrollierte Studie zu den Effekten transkranieller Gleichstromstimulation an sechs Patienten ein Jahr nach stattgehabtem Schlaganfall. Gemeinsam war dem Patientenkollektiv eine motorische Parese des Armes. Durch eine 20-minütige anodale tDCS konnte eine Verbesserung der feinmotorischen Funktion der paretischen Hand während der Stimulation und bis zu 25 Minuten nach Stimulation nachgewiesen werden. Diese Ergebnisse deuten darauf hin, dass transkranielle Gleichstromstimulation eine sinnvolle zusätzliche Therapiemethode in der Neurorehabilitation sein könnte. Ebenso konnten positive Effekte durch tDCS in der Schmerzforschung aufgedeckt werden. Eine doppelblinde, plazebokontrollierte 
Studie an 32 Patienten mit Fibromyalgie ergab eine signifikante Schmerzreduktion nach einer 20-minütigen tDCS von $2 \mathrm{~mA}$ über dem motorischen Kortex an fünf aufeinander folgenden Tagen, welche sogar bis zu zwei Wochen nach Ende der Stimulation anhielt (Fregni et al 2006d). Darüber hinaus konnte tDCS über dem visuellen Kortex von Migränepatienten zu einer signifikanten Schmerzreduktion führen (Antal et al. 2011). Schließlich zeigte auch eine Studie an 17 Patienten mit Morbus Parkinson, dass eine 20-minütige anodale tDCS über dem motorischen Kortex zu einer verbesserten motorischen Funktion führte, wie es anhand der Unified Parkinson's Disease Rating Scale (UPDRS), einer Reaktionszeitaufgabe und anhand des Purdue Pegboard Test (PPT) nachgewiesen wurde (Fregni et al. 2006e).

Ebenso fand die transkranielle Gleichstromstimulation bereits Anwendung bei Patienten mit Tinnitus. In einer offenen Studie an 543 Patienten mit chronischem Tinnitus konnte bei ca. einem Drittel der Patienten mit 20-minütiger bilateraler tDCS von 1,5 mA über dem dorsolateralen präfrontalen Kortex mit Positionierung der Anode über F4 (rechter DLPFC) und der Kathode über F3 (linker DLPFC) eine Reduktion des ausgelösten Stress und der Intensität des Geräuschs erreicht werden (Vanneste et al. 2010).

Schließlich wurden auch die Auswirkungen transkranieller Gleichstromstimulation auf die Gedächtnisleistungen von Alzheimer-Patienten untersucht: Dabei zeigten Boggio et al. 2009 an einer Gruppe von 10 Alzheimer-Patienten, dass drei Sitzungen einer 30-minütigen anodalen tDCS von $2 \mathrm{~mA}$ über dem DLPFC und dem links temporalen Kortex zu einer besseren visuellen Erinnerung im Vergleich zur Plazebo-Stimulation führten. Neben dem Einsatz als weitere Therapieoption bei der größten Gruppe der neurologischen und psychiatrischen Krankheiten der westlichen Industrienationen wird tDCS ebenfalls in neurophysiologischen Verhaltensstudien angewandt.

\subsubsection{Studien zur Lern- und Gedächtnisformierung}

Ein besonders spannendes und seit Jahrzehnten untersuchtes Feld sind Mechanismen von Lern- und Gedächtnisleistungen des menschlichen Gehirns. Auch die transkranielle Gleichstromstimulation wurde in diversen Studien zu Lernund Gedächtnisleistungen angewandt. Unter anderem konnte dabei gezeigt werden, dass anodale und kathodale tDCS visuell-motorische Koordinationsaufgaben an jungen, gesunden Probanden positiv beeinflussen können (Antal et al. 2004b, c). Außerdem konnte anodale tDCS über dem dorsolateralen präfrontalen Kortex an 
gesunden Probanden verbesserte Leistungen des verbalen Arbeitsgedächtnisses erzeugen, die sogar über die Stimulation hinaus nachweisbar waren (Fregni et al. 2005; Ohn et al. 2008). Des Weiteren konnte durch 20-minütige anodale tDCS ein schnelleres, effizienteres Lernen neuer Sprachvokabeln und eine verbesserte verbale Sprachflüssigkeit erzielt werden (Flöel et al. 2008, lyer et al. 2005). Ebenso konnte eine verbesserte motorische Lernleistung durch anodale Stimulation erreicht werden (Nitsche et al. 2003d; Reis et al. 2009; Stagg und Nitsche 2011). Die Ergebnisse der eben beschriebenen Experimente legen nahe, dass tDCS und Lernen durch ähnliche Prozesse im Gehirn agieren.

Einige dieser Mechanismen hat man in den letzten Jahrzehnten bereits aufdecken können:

Unumstritten ist die Tatsache, dass der Erwerb motorischer Fähigkeiten oder aber das Akquirieren von neuem Wissen und die anschließende Speicherung dieser Informationen plastische Veränderungen innerhalb des Gehirns hervorrufen, welche sich in der Veränderung von synaptischen Verknüpfungen widerspiegeln.

Die Fähigkeit des menschlichen Gehirns, sich seiner Umwelt anzupassen und seine synaptischen Verknüpfungen je nach Stärke und Frequenz der übertragenen elektrischen Signale umzustrukturieren, bezeichnet man als Neuroplastizität.

Eine spezifische Plastizitätsform spielt bei Lern- und Erinnerungsvorgängen eine wichtige Rolle. Diese ist die Langzeitpotenzierung. Die Ursprünge der Forschung von synaptischer Plastizität liegen beim Neuropsychologen Donald Hebb, der in seiner bedeutenden Publikation von 1949 "The Organization of Behaviour" postulierte, dass es zwischen zwei Neuronen zu einer Verstärkung der synaptischen Verbindung kommt, wenn ein Neuron über sein Axon ein anderes Neuron wiederholt erregt und in diesem Zielneuron zur Auslösung von Aktionspotentialen führt. Die Entdeckung des Phänomens der Langzeitpotenzierung erfolgte dann schließlich Ende der 60er und Anfang der 70er Jahre durch Bliss und Lomo (Bliss und Lomo 1973). Sie zeigten im Tierversuch, dass die hochfrequente, repetitive Stimulation des Hippokampus von anästhesierten Hasen zu einer länger als 30 Minuten anhaltenden Steigerung der synaptischen Übertragungsrate führte. Seitdem haben zahlreiche weitere Studien die Induktion von LTP in verschieden Kortexarealen von Tieren (Castro-Alamancos et al. 1995; Hess und Donoghue 1994; Nosten-Bertrand et al. 1996; Urban et al. 1996) und 
ebenso in humanen Hippokampuspräparaten (Beck et al. 2000) zeigen können (Bliss et al. 2003; Cooke und Bliss 2006).

Die Beteiligung von LTP an Lern- und Erinnerungsvorgängen gilt durch die Resultate vieler Studien als gesichert (Bliss und Collingridge 1993; Martin et al. 2000; RioultPedotti et al. 1998, 2000; Ziemann et al. 2004). Auf molekularer Ebene kennt man aus Tierversuchen an Hippokampuspräparaten die zugrundeliegenden Abläufe, welche zur Auslösung und Aufrechterhaltung synaptischer Plastizitätsveränderungen, die länger als 30 Minuten anhalten, vonnöten sind. Das Phänomen LTP wird in eine Früh- (e-LTP) und eine Spätphase (I-LTP) unterteilt. Darüber hinaus weiß man aus diesen Experimenten, dass eine erregbarkeitssteigernde synaptische Plastizitätsveränderung, die bis zu einer Stunde nach Stimulation nachweisbar ist, von einer erhöhten NMDA-Rezeptor-Aktivität abhängt, wohingegen länger anhaltende Nacheffekte, die über mehrere Stunden oder Tage anhalten, neue Proteinsynthese benötigen (Frey et al 1993; Huang YY et al 2004; Reymann und Frey 2007).

Vor allem hochfrequente, tetanische Stimulationsformen konnten im Tierexperiment die Ausbildung von LTP induzieren.

Neuere Studien zeigen, dass auch nicht-invasive, neuromodulatorische Stimulationsformen am Menschen eine LTP-ähnliche Plastizität bewirken können.

Repetitive transkranielle Magnetstimulation (rTMS), gepaarte assoziative Stimulation (PAS), Theta-Burst-Stimulation (TBS) und auch die transkranielle Gleichstromstimulation (tDCS) sind im Stande, e-LTP im menschlichen Kortex zu induzieren (Huang YZ et al. 2007; Nitsche und Paulus 2000, 2001; Stefan et al. 2000,2002; Ziemann und Siebner 2008). Gemeinsam ist all diesen Stimulationsformen - konkordant zu tierexperimentellen Ergebnissen - die Tatsache, dass sie zur Produktion e-LTP-ähnlicher Plastizität verstärkte NMDA-RezeptorAktivität benötigen.

Im Gegensatz zur Frühphase der Langzeitpotenzierung konnte keine der vorgestellten Stimulationsformen im menschlichen Kortex I-LTP erzeugen.

Tierexperimentell erfordert diese Art der Neuroplastizität - ebenso wie das Speichern und Reaktivieren von Gedächtnisinhalten als Langzeiterinnerung - neue Proteinsynthese. Daher wird I-LTP stets als neuronales Modell für Langzeiterinnerungen verwendet (Costa-Mattioli et al. 2009; Sossin 2008). 
Interessanterweise haben verschiedene Studien im Tier- und Humanversuch herausgefunden, dass wiederholtes Lernen in kleineren Einheiten - im Gegensatz zu einer allumfassenden, einmaligen großen Trainigseinheit - bessere Gedächtnisleistungen erzeugt (Kim M et al. 2010; Kornell et al. 2010; Xue et al. 2011).

Äquivalent wird I-LTP im Tierexperiment durch den Einsatz repetitiver, mehrfacher Stimulationen, die üblicherweise durch eine Pause von 5-10 Minuten voneinander getrennt sind, ausgelöst. Eine einmalige tetanische Stimulation führt lediglich zur Induktion einer e-LTP (Costa-Mattioli et al. 2009; Gong et al. 2011).

Diese Erkenntnisse machen den Einsatz von repetitiven, nicht-invasiven Stimulationen mit einem freien Intervall von wenigen Minuten zur Produktion von sehr langanhaltenden kortikalen Exzitabilitätsveränderungen im Humanexperiment zu einem interessanten Untersuchungsthema.

\subsubsection{Bisherige Studien zu repetitiver tDCS}

Die meisten Studien zur transkraniellen Gleichstromstimulation befassten sich mit der Beeinflussung der Aktivität und Erregbarkeit tierischer und menschlicher Gehirne durch kontinuierliche Stimulation. Dabei wurden die Polarität des stimulierten Areals, die Stromstärke (1-2 mA), die Größe der verwendeten Elektrode $\left(25-35 \mathrm{~cm}^{2}\right)$, sowie die Stimulationsdauer in unterschiedlicher Weise variiert. Dies sind auch die Faktoren, welche als bedeutende Einflussgrößen für die Art, Dauer und Intensität der produzierten Nacheffekte stets genannt werden (Nitsche et al. 2008).

Nur wenige Studien haben sich mit der Frage beschäftigt, welche physiologische Auswirkung eine repetitive Gleichstromstimulation für das Erregbarkeitsniveau innerhalb des menschlichen Gehirns hat.

Eine dieser Studien wurde parallel zum Experiment dieser Arbeit durchgeführt und untersuchte die Effekte repetitiver kathodaler Gleichstromstimulation. Der Einsatz einer zweiten Stimulation zum Zeitpunkt der Nacheffekte der ersten Stimulation bei einer Pause von wenigen Minuten führte zu einer effektiveren und elongierten Erregbarkeitsverminderung des motorischen Kortex bis zur 120. Minute nach tDCS. Dem gegenüber gestellt ließ eine Pause von 3 Stunden oder 24 Stunden die erregbarkeitsvermindernden Effekte bis zur 60. Minute verschwinden, mit einer darauffolgend wieder einsetzenden Inhibition. Die Ergebnisse der Studie deuten darauf hin, dass repetitive Stimulationsformen mit einem dazwischen liegenden 
Intervall von wenigen Minuten in der Lage sind, länger andauernde und intensivere Nacheffekte zu erzeugen (Monte-Silva et al. 2010).

In einem anderen Experiment resultierte repetitive tDCS von wenigen Minuten, die nur kurz anhaltende Nacheffekte erzeugt, mit Pausen zwischen einer Minute bis zu 30 Minuten teilweise in der Ausbildung von homöostatischer Plastizität (Fricke et al. 2011).

Zusätzlich zu den eben erwähnten Studien, die sich mit den zugrundeliegenden Mechanismen von repetitiver tDCS beschäftigen, gibt es einige repetitive Stimulationsformen, die bereits in der klinischen Anwendung an Patienten getestet werden. So zum Beispiel in der von Fregni et al. (2006b) veröffentlichten Studie an 10 Patienten mit Major Depression, bei denen eine tägliche 20-minütige anodale tDCS an fünf aufeineinander folgenden Tagen zu einer signifikanten Verminderung depressiver Symptome führte.

\subsection{Zielsetzung}

Meine bisherigen Ausführungen fassen den aktuellen Stand der Forschung zur transkraniellen Gleichstromstimulation zusammen. Es sollte nochmals festgehalten werden, dass die tDCS eine relativ einfach anzuwendende, nicht-invasive und risikoarme Methode der elektrischen Stimulation am menschlichen Gehirn darstellt, die in Pilotstudien zur klinischen Anwendung an neuropsychiatrischen Patienten kommt und einige Erfolge zur Therapieoptimierung liefern konnte. Die Ergebnisse der letzten Jahrzehnte wecken die Hoffnung, dass sich diese Methode möglicherweise im klinischen Alltag der Neurologie, Psychiatrie und anderer medizinischer Disziplinen als modifizierendes, neues Behandlungsverfahren etablieren könnte. Doch dafür werden Stimulationsbedingungen benötigt, die über einen möglichst langen Zeitraum stabile kortikale Erregbarkeitsveränderungen erzeugen - dies könnte eventuell durch repetitive Stimulationen erreicht werden, weshalb ich mich in meiner Dissertation mit der Fragestellung der Veränderungen neuronaler Erregbarkeit des motorischen Kortex des Menschen durch repetitive anodale Gleichstromstimulation beschäftige. 
Folgende Grundlagen dienten mir zur Formulierung der Hypothesen dieser Dissertation:

Kontinuierliche anodale tDCS über dem Motorkortex resultiert in einer Steigerung der kortikalen Erregbarkeit, die bis zu einer Stunde nach Stimulationsende nachweisbar ist (Nitsche und Paulus 2000, 2001).

Bei der Anwendung von repetitiven Stimulationen mit relativ kurzen Pausen von einigen Minuten hat die zuvor erwähnte Parallelstudie für kathodale tDCS effektivere und länger andauernde Erregbarkeitssenkungen des kortikalen Aktivitätslevels im Vergleich zu kontinuierlicher kathodaler tDCS dokumentieren können (Monte-Silva et al. 2010) .

Weiterhin wurde tierexperimentell innerhalb der Forschung zu Lern- und Gedächtnisleistungen nachgewiesen, dass repetitive hochfrequente Stimulationsformen, welche im Abstand von wenigen Minuten appliziert wurden, langanhaltende I-LTP-ähnliche Plastizität induzieren konnten (Bengtson et al. 2010; Frey et al. 1993; Huang YY et al. 1996).

Anhand dieser Ausgangsbedingungen stellt sich mir die Frage, ob repetitive anodale tDCS mit stimulationsfreien Zeiten von wenigen Minuten in der Lage ist, länger anhaltende ( $>1$ Stunde) und effektivere kortikale Erregbarkeitssteigerungen zu induzieren, und, genauer gesagt, zur Formierung I-LTP-ähnlicher Plastizität im Menschen geeignet ist. Mit dieser Frage werde ich mich im Verlauf der Arbeit gezielt auseinandersetzen. Um die repetitive tDCS umfassender zu betrachten, werde ich nicht nur ein stimulationsfreies Intervall von wenigen Minuten, welches sich tierexperimentell am geeignetsten zur Auslösung verstärkter synaptischer Plastizität im Sinne eines I-LTP erwiesen hat, sondern auch eine Pause mehrerer Stunden zwischen zwei Stimulationen betrachten und diese in Gegenüberstellung zu einer kontinuierlichen anodalen tDCS derselben Gesamtdauer, sowie zu der bereits untersuchten kontinuierlichen anodalen tDCS von 13 Minuten in Beziehung setzen. Dadurch möchte ich ermitteln, ob alleinig die Dauer der Stimulation oder aber der Zeitpunkt, zu dem die verschiedenen Stimulationen erfolgen, für die Effekte der repetitiven tDCS von Bedeutung ist. 
Schlussendlich führen mich diese Basiserkenntnisse zu folgenden Hypothesen, die ich im Verlauf der Arbeit erörtern werde:

1. Repetitive 13-minütige anodale transkranielle Gleichstromstimulation mit einer Pause von wenigen Minuten resultiert - in Anlehnung an tierexperimentelle Untersuchungen - in der Induktion von I-LTP im humanen Motorkortex.

2. Eine Verlängerung der anodalen Stimulationsdauer führt - wie in früheren Studien kontinuierlicher anodaler tDCS - zu einer Verlängerung der erregbarkeitssteigernden Nacheffekte innerhalb des humanen Motorkortex.

3. Ein stimulationsfreies Intervall von mehreren Stunden resultiert in ähnlichen kortikalen Erregbarkeitsveränderungen wie einfache kontinuierliche anodale tDCS von 13 Minuten, da die beiden Stimulationen durch den großen zeitlichen Abstand unabhängig voneinander erfolgen. 


\section{Material und Methoden}

\section{1 Übersicht}

In diesem Teil werde ich die angewandten Methoden zur Untersuchung der Effekte repetitiver anodaler Gleichstromstimulation auf die kortikale Erregbarkeit des motorischen Kortex skizzieren. Zunächst werde ich das beteiligte Probandenkollektiv beleuchten, die Funktionsweisen der angewandten Methoden der nicht-invasiven transkraniellen Stimulationsformen (TMS und tDCS) beschreiben und ihre Anwendung am Probanden schildern.

Außerdem werde ich das Medikament Flunarizin mit seinem Wirkmechanismus und seinen Nebenwirkungen beschreiben, da dieses in einem Kontrollexperiment (Experiment 2) zum Einsatz kam, um die ursächlichen Mechanismen der Nacheffekte kontinuierlicher 26-minütiger anodaler tDCS des 1. Experiments auf die kortikale Erregbarkeit genauer zu analysieren

Schließlich werde ich die Versuchsdurchführung der Experimente 1 und 2 darstellen und die angewandte Statistik zur Auswertung der Daten erläutern.

\subsection{Probanden}

An der Studie zu repetitiver anodaler Gleichstromstimulation nahmen 15 junge, gesunde Probanden teil (Altersspanne: 20.-34. Lebensjahr, Durchschnittsalter: 25,53 Jahre +/- 3,6; 9 weibliche, 6 männliche Probanden; Studenten an der Georg-AugustUniversität Göttingen). Am Kontrollexperiment mit pharmakologischer Intervention durch das Medikament Flunarizin beteiligten sich 5 dieser 15 Probanden (Durchschnittsalter: 24,6 Jahre +/- 3,1; 3 weibliche, 2 männliche Probanden). Zunächst wurde jeder Teilnehmer ausführlich über die Zielsetzung der Experimente, die angewandten Methoden (TMS, tDCS, das Medikament Flunarizin), den Versuchsablauf und die möglichen Nebenwirkungen aufgeklärt. Bei Vorhandensein einer der folgenden Kontraindikationen zur Studienteilnahme, wie das Tragen eines Herzschrittmachers, Metallimplantate im Kopfbereich, bekannte zerebrale Ischämien, schwerwiegende neurologische, internistische oder psychiatrische Erkrankungen, bereits aufgetretene epileptische Anfälle, bestehende Schwangerschaft oder Stillperiode, Medikamenten-, Alkohol-, oder Drogenabhängigkeit sowie ein Alter 
jünger als 18 Jahre oder älter als 75 Jahre, wurde die betreffende Person von der Studienteilnahme ausgeschlossen. Jeder Proband gab nach dem persönlichen Aufklärungsgespräch seine schriftliche Zustimmung zur Versuchsteilnahme. Vor Beginn der Studie erklärte die zuständige Ethikkommission der Universität Göttingen ihr Einverständnis zur Durchführung der Studie (Ethik-Antrags-Nr.: 15/4/99).

\subsection{TMS über dem motorischen Kortex}

Um die Veränderungen der Aktivität und Erregbarkeit der durch tDCS stimulierten Neuronenpopulation nachzuweisen, wurde die transkranielle Magnetstimulation angewandt. Die TMS ist eine bekannte und bewährte Methode, die bereits mehrfach in Experimenten dieser Art zum Einsatz gekommen ist (Nitsche und Paulus 2000, 2001; Nitsche et al. 2003b).

Dabei werden von einer Spule kurze, magnetische Impulse über dem zu stimulierenden kortikalen Areal durch die Kopfhaut appliziert. Hierdurch wird ein elektrischer Stromfluss im Gehirn ausgelöst, der zur Depolarisation und anschließenden Auslösung von Aktionspotentialen an der Nervenzelle führt. Wird diese Stimulation über dem motorischen Kortex durchgeführt, werden hierdurch an den Muskeln, deren kortikales Repräsentationsgebiet stimuliert wird, motorisch evozierte Potentiale (MEP) ausgelöst. Deren Amplitude ist ein globales Maß der kortikospinalen Erregbarkeit (Rothwell 1993). Die Amplitudenhöhe der MEPs wurde vor und nach der Gleichstromstimulation miteinander verglichen, um durch tDCS ausgelöste neuroplastische Erregbarkeitsveränderungen messbar zu machen.

Der Magnetstimulator Magstim 200 (Magstim Company, Whiteland, Dyfed, UK) gab über eine achtförmige Spule (Durchmesser einer Windung $70 \mathrm{~mm}$, Spitzenmagnetfeld 2,2 Tesla) Einzelmagnetimpulse ab, um jenes Areal des linken motorischen Kortex zu stimulieren, welches den rechten Musculus abductor digiti minimi (ADM) repräsentiert. Für eine optimale Ausbreitung der Impulse wurde die Magnetspule möglichst tangential zum Schädel in einem $45^{\circ}$-Winkel zur Mittellinie gehalten. Die Spulenposition, welche konstant die höchsten MEPs lieferte, wurde als optimale Spulenposition definiert. Die Aktivität des Zielmuskels (ADM) wurde über Oberflächenelektroden am Muskelbauch und seiner Sehne registriert.

Diese Signale wurden mit einer Zeitkonstante von $10 \mathrm{~ms}$ und einem Bandpassfilter mit der Grenzfrequenz von 2,5 kHz erfasst. Danach wurden die Daten digitalisiert 
und im Laborcomputer mittels Signal Software und CED 1401 Hardware (Cambridge Electronic Design, Cambridge, UK) dargestellt.

Die Intensität der Magnetstimulation wurde so angepasst, dass die Amplituden der MEPs vor der tDCS im Durchschnitt $1 \mathrm{mV}$ betrugen, und im Verlauf der Experimente nicht verändert.

\section{4 tDCS über dem motorischen Kortex}

Die transkranielle Gleichstromstimulation (tDCS) ist die Methode, mit welcher ich mich vorrangig in meiner Arbeit beschäftige und deren Einfluss auf die Neuroplastizität des menschlichen Gehirns bei repetitiver Anwendung in der vorliegenden Arbeit untersucht wurde.

Bei der tDCS wird ein schwacher Gleichstrom über die Kopfhaut appliziert. Dieser führt im motorischen Kortex zu unterschwelligen Polaritätsverschiebungen des Ruhememembranpotentials der Neuronen und zu einer Veränderung ihrer Erregbarkeit. Aktionspotentiale werden im Gegensatz zur TMS dabei nicht ausgelöst. In meinem Experiment erfolgte die transkranielle Gleichstromstimulation durch einen batteriebetriebenen Gleichstromstimulator (Schneider Electronic, Gleichen Deutschland), welcher eine maximale Stromstärke von $2 \mathrm{~mA}$ generierte. Die Leitung des Stroms erfolgte über zwei mit 0,9\% NaCl-Lösung befeuchtete und $35 \mathrm{~cm}^{2}$ große Oberflächen-Schwammelektroden, die durch elastische Bänder an der Kopfhaut der Probanden fixiert wurden. Die Elektroden wurden an dem zuvor durch TMS ermittelten Repräsentationsort des rechten ADM über dem linken motorischen Kortex und oberhalb der kontralateralen Orbita positioniert.

In vorherigen Studien hatten sich diese Elektrodenpositionen als optimal erwiesen, um Erregbarkeitsveränderungen des motorischen Kortex hervorzurufen (Nitsche und Paulus 2001).

Als anodaler Pol wurde diejenige Elektrode definiert, die über dem motorischen Kortex platziert war. Es wurde eine anodale Gleichstromstimulation dieses Areals von 13 Minuten (bzw. 26 Minuten - siehe Stimulationsbedingungen Seite -20- und Versuchsdurchführung Seite -53-) mit einer Stromstärke von $1 \mathrm{~mA}$ durchgeführt. Frühere Studien haben gezeigt, dass diese Stimulationsdauer zu einer gesteigerten Erregbarkeit des motorischen Kortex führt, welche länger als 1 Stunde nach der Stimulation nachweisbar ist (Nitsche und Paulus 2001). Während der Stimulation 
wurden die Stromstärke, die Stromspannung und das Befinden des Probanden kontinuierlich überwacht. Viele Teilnehmer registrierten den Stromfluss als Jucken oder unangenehmes Brennen, das durch ein erneutes Befeuchten der Schwammelektroden mit $\mathrm{NaCl}$ schnell behoben werden konnte.

\subsection{Medikation mit Flunarizin}

Da eine Beteiligung von Kalzium als Grund für die Erregbarkeitsverminderung des motorischen Kortex nach 26-minütiger anodaler transkranieller Gleichstromstimulation vermutet wurde, untersuchte ich diese These in einem Kontrollexperiment durch die Applikation von $10 \mathrm{mg}$ Flunarizin.

Zwei Stunden vor Beginn der anodalen Gleichstromstimulation im Experiment 2 (Kontrollexperiment) nahmen die Probanden $10 \mathrm{mg}$ Flunarizin (FLU) p.o. zu sich. Flunarizin ist ein Kalzium-Kanal-Antagonist, der zentralnervöse Wirkungen zeigt und in der Klinik zur Behandlung von Schwindelerkrankungen zum Einsatz kommt.

Unter dieser Konzentration des Medikaments sind deutliche Beeinflussungen des zentralen Nervensystems beschrieben worden (Louis und Spierings 1982; Nitsche et al. 2003a, Stoica und Enulescu 1993). Aus Studien ist bekannt, dass eine maximale Plasmakonzentration von Flunarizin zwei Stunden nach oraler Einnahme erreicht wird (Holmes et al. 1984).

\subsection{Versuchsdurchführungen}

\subsubsection{Experiment 1}

Jede Sitzung des Experiments begann damit, dass der Proband eine bequeme und entspannte Position auf dem Liegestuhl des Labors einnahm. Nach einigen Minuten der Entspannung wurde mit der Vorbereitung für die Messungen begonnen. Dafür wurden Oberflächenelektroden an der rechten Hand im Bereich des Muskelbauches und der Sehne des ADM mit Klebeband fixiert und die Erdungselektrode am selbigen Handgelenk angebracht. Schließlich wurden die Kopfstützen in eine für den Probanden angenehme Position gebracht und bei Bedarf die Höhe des Liegestuhls verändert. Nachdem diese Vorbereitungen abgeschlossen waren, wurde der kortikale Repräsentationsort des rechten ADM mittels transkranieller Magnetstimulation 
ermittelt. Die Spulenposition, welche konstant die höchsten MEPs lieferte, wurde als Referenzpunkt festgelegt und mit einem wasserfesten Stift markiert. Die Intensität der TMS wurde so angepasst, dass die Amplitudenhöhe der MEPs im Durchschnitt 1 $\mathrm{mV}(+/-0,2 \mathrm{mV})$ ergab. Mit diesen Einstellungen konnte die Ausgangsmessung vor tDCS (Baseline) in Form von 25 MEPs über dem markierten Repräsentationspunkt in der zuvor bestimmten TMS-Intensität mit einer Frequenz von $0,25 \mathrm{~Hz}$ aufgezeichnet werden. Die Nucursor-Software des Computers berechnete den Mittelwert der Amplitudenhöhe der Baseline. Die Arbeitsschritte bis zur Aufzeichnung der Baseline nahmen ca. 30 Minuten in Anspruch. Danach wurde die anodale transkranielle Gleichstromstimulation durch den DC-Stimulator mit einer Stromstärke von $1 \mathrm{~mA}$ durchgeführt. Die anodale Elektrode wurde über dem linken Motorkortex an der zuvor markierten Stelle befestigt, während die andere Elektrode an der Stirn der kontralateralen Orbita positioniert wurde.

Die Stimulationsdauer betrug 13 Minuten oder 26 Minuten. Die Stimulationsform war kontinuierlich oder repetitiv mit einer variablen Pause.

\section{Stimulationsbedingungen des Experiments 1:}

\section{a) kontinuierliche Gleichstromstimulation}

1. Sitzung: $13 \mathrm{~min}$ anodale Gleichstromstimulation

2. Sitzung: $26 \mathrm{~min}$ anodale Gleichstromstimulation

b) repetitive Gleichstromstimulation mit kurzen Pausen

3. Sitzung: 13 min anodale Gleichstromstimulation - 3 min Pause 13 min anodale Gleichstromstimulation

4. Sitzung: $13 \mathrm{~min}$ anodale Gleichstromstimulation - 20 min Pause 13 min anodale Gleichstromstimulation

c) repetitive Gleichstromstimulation mit langen Pausen

5. Sitzung: 13 min anodale Gleichstromstimulation - 3 h Pause 13 min anodale Gleichstromstimulation

6. Sitzung: $13 \mathrm{~min}$ anodale Gleichstromstimulation - $24 \mathrm{~h}$ Pause 13 min anodale Gleichstromstimulation 
Durch eine Stoppuhr wurde die Einhaltung der vorgegebenen Stimulations- und Pausenzeiten sichergestellt.

Sofort nach Ende der tDCS wurden die Stimulationselektroden entfernt und 25 MEPs in der Intensität der Baseline mittels TMS am Repräsentationsort des rechten ADM über dem linken motorischen Kortex abgeleitet. Diese Messungen wurden bis zur 30. Minute nach Stimulationsende alle 5 Minuten wiederholt. Danach wurden jeweils weitere 25 MEPs in der 60. Minute, der 90. Minute und in der 120. Minute nach anodaler Gleichstromstimulation aufgezeichnet.

Die Erregbarkeitsveränderungen über dem Motorkortex wurden am selben Abend, am nächsten Morgen, sowie am Mittag und Abend des Folgetages nach der letzten anodalen tDCS durch MEP-Messungen überwacht. Eine Ausnahme davon stellte die Sitzung kontinuierlicher 13-minütiger anodaler tDCS dar, bei der die Nachmessungen nur bis zum Abend des Stimulationstages durchgeführt wurden, weil bei dieser Stimulation keine länger anhaltenden Nacheffekte zu erwarten waren (Nitsche und Paulus 2000, 2001).

Das zeitliche Intervall zwischen den Nachmessungen betrug jeweils mindestens 3 Stunden.

Zwischen 2 Sitzungen musste ein zeitlicher Abstand von mindestens 7 Tagen bestehen, um Interaktionen zwischen den verschieden Stimulationskonditionen zu vermeiden. Die Reihenfolge der unterschiedlichen Stimulationsbedingungen erfolgte randomisiert.

\subsubsection{Experiment 2}

Das Kontrollexperiment wurde in Anlehnung an die Sitzung kontinuierlicher 26minütiger anodaler Gleichstromstimulation durchgeführt. Die Laborbedingungen, die verwendeten Programme und Materialien differierten nicht vom Experiment 1. Nach der Aufzeichnung der MEP-Ausgangsmessung vor tDCS nahmen die Probanden 10 mg Flunarizin p.o. zu sich. Nach einer zweistündigen Pause wurde die zuvor bestimmte Baseline (Baseline 2) erneut überprüft, um Veränderungen der kortikalen Erregbarkeit durch das Medikament zu erfassen, und falls nötig so angepasst (Baseline 3), dass der Mittelwert der Amplitudenhöhe der 25 MEPs bei $1 \mathrm{mV}$ lag. 
Danach erfolgte die 26-minütige anodale Gleichstromstimulation mit anschließender Überprüfung der kortikalen Erregbarkeitsveränderungen mittels TMS bis zur 120 . Minute wie im Experiment 1 bereits beschrieben.

(Siehe auch Abbildung 5 im Anhangsverzeichnis; Seite -53-)

\subsection{Datenauswertung und Statistik}

Zur Auswertung der im Experiment erhobenen Messwerte wurden jeweils die Mittelwerte der MEPs der Ausgangsmessung vor tDCS (Baseline) und der 11-14 TMS-Messungen nach transkranieller Gleichstromstimulation mit oder ohne medikamentösen Einfluss durch Flunarizin bestimmt (bei jeder Kondition jeweils 25 MEPs pro Messzeitpunkt). Die Messwerte nach tDCS wurden intraindividuell als Quotient der MEPs der Baseline angegeben.

In Experiment 1 wurden diese Ergebnisse mit einer Varianzanalyse (ANOVA) für Messwiederholungen unter Berücksichtigung der abhängigen Variable „Amplitude der MEPs“, sowie der Messwiederholungs-Faktoren „Zeitverlauf“ und „tDCSStimulationsbedingung" berechnet.

Im Experiment 2 wurde eine Messwiederholungs-ANOVA mit den Faktoren „Zeitverlauf" und „Medikation" durchgeführt, um die Bedingungen mit oder ohne Flunarizineinnahme auf eine signifikante Abweichung hin zu überprüfen.

Weiterhin wurde der Fisher-LSD-Test angewandt, um festzustellen, ob sich die Amplituden der MEPs zwischen den verschiedenen tDCS-Bedingungen, außerdem vor und nach der Gleichstromstimulation oder aber in Gegenüberstellung von wiederholter und kontinuierlicher Gleichstromstimulation (13 min-0-0-Bedingung) signifikant voneinander unterscheiden. Allen Analysen lag ein kritischer $p$-Wert von $\leq$ 0,05 zugrunde. Es wurden jeweils die Mittelwerte mit dazugehöriger Standardabweichung bestimmt.

Weitere statistische Werkzeuge waren der Mauchly-Test zur Überprüfung der Sphärizität der den ANOVAs zugrunde liegenden Daten, sowie die GreenhouseGeisser-Korrektur, die im Bedarfsfall eingesetzt wurden. 


\section{Ergebnisse}

\subsection{Ausgangssituation}

In den zwei zuvor dargestellten Experimenten wurden die Effekte von repetitiver anodaler Gleichstromstimulation auf die Erregbarkeitsveränderungen des motorischen Kortex untersucht. Die 6 Sitzungen des Experiments 1 umfassten zwei Stimulationsbedingungen mit kontinuierlicher anodaler Gleichstromstimulation von 13 Minuten bzw. 26 Minuten, sowie 4 Sitzungen mit wiederholter anodaler Gleichstromstimulation von jeweils 13 Minuten, die durch unterschiedlich lange Pausen voneinander separiert waren. Zusätzlich überprüften wir im Experiment 2 die Beeinflussung der kortikalen Erregbarkeitsveränderung von 26-minütiger kontinuierlicher anodaler Gleichstromstimulation durch den Kalziumkanalblocker Flunarizin.

Im Folgenden werde ich zunächst die Ergebnisse des Experiments 1 mit den dazugehörigen Tabellen und Graphiken der einzelnen Stimulationsbedingungen darstellen. Im Anschluss daran werde ich die Resultate des zweiten Experiments in gleicher Weise erläutern und schließlich eine Zusammenfassung des Ergebnisteils präsentieren.

\subsection{Ergebnisse des Experiments 1}

Die Intensität der TMS, welche zum Erreichen einer MEP-Amplitude von $1 \mathrm{mV}$ notwendig war, sowie die MEP-Amplitude der Baselinemessungen unterschieden sich nicht zwischen den 6 tDCS-Bedingungen (siehe Tabelle 1) - bis auf einen leichten, aber dennoch signifikanten Unterschied zwischen der 13 min-20 min-13 min-Stimulationsbedingung auf der einen Seite und den 13 min-0-13 min- und 13 min-24 h-13 min-Stimulationsbedingungen auf der anderen Seite. Diese Abweichungen hatten keinen Einfluss auf die Ergebnisse der Hauptanalyse. Die Stimulationsintensität der TMS (als prozentualer Anteil der maximalen Leistung des TMS-Stimulators angegeben [\%MSO]), welche in der Lage war, eine MEP-Amplitude von ca. $1 \mathrm{mV}$ zu erzeugen, unterschied sich nicht zwischen den einzelnen Stimulationsformen des Experiments 1 (Fisher-LSD-Test: $p>0,05)$ (siehe Tabelle 1). 


\section{Tabelle 1: Baseline MEP-Amplitudenhöhe \& TMS-Intensität Experiment 1}

Für jede der 6 Stimulationsbedingungen des 1. Experiments kontinuierlicher und repetitiver 13-minütiger anodaler tDCS mit unterschiedlichen Pausen ist die dazugehörige Amplitudenhöhe der MEP-Ausgangsmessung vor tDCS (Baseline) in Millivolt $(\mathrm{mV})$ und die TMS-Intensität in \% der maximalen Stimulatorleistung dargestellt. Die Pausen zwischen den repetitiven Stimulationen sind in Minuten (min) oder Stunden (h) angegeben. Alle Messwerte entsprechen Mittelwerten mit dazugehöriger Standardabweichung.

\begin{tabular}{|l|l|l|}
\hline $\begin{array}{l}\text { Stimulationsform: tDCS } \\
\text { in } \text { min - Pause in } \mathbf{m i n} / \mathbf{h}- \\
\text { tDCS in } \text { min }\end{array}$ & $\begin{array}{l}\text { MEPs der Baseline in } \\
\mathbf{m V}\end{array}$ & $\begin{array}{l}\text { TMS-Intensitäten in \% } \\
\text { der maximalen } \\
\text { Stimulatorleistung }\end{array}$ \\
\hline $13-0-0$ & $0,947+/-0,10$ & $45,7+/-0,07$ \\
\hline $13-0-13$ & $1,003+/-0,10$ & $43,9+/-0,07$ \\
\hline $13-3 \mathrm{~min}-13$ & $0,947+/-0,13$ & $45,1+/-0,06$ \\
\hline $13-20 \mathrm{~min}-13$ & $0,890+/-0,10$ & $44,3+/-0,08$ \\
\hline $13-3 \mathrm{~h}-13$ & $0,951+/-0,13$ & $44,5+/-0,06$ \\
\hline $13-24 \mathrm{~h}-13$ & $0,999+/-0,12$ & $44,3+/-0,06$ \\
\hline
\end{tabular}

Die ANOVA, die für das Hauptexperiment (Experiment 1) durchgeführt wurde, ergab einen signifikanten Haupteffekt des Faktors „Gleichstromstimulationsbedingung“ (FWert: 4,239; Df: 5; p-Wert: 0,002), sowie eine signifikante Interaktion zwischen den Faktoren „Gleichstromstimulationsbedingung“ und „Zeit“ (F-Wert: 1,638; Df: 11; pWert: 0,003) (siehe Tabelle 2).

\section{Tabelle 2: Ergebnisse der ANOVA des Experiments 1}

Daten der Messwiederholungs-ANOVA des Experiments 1 mit der abhängigen Variable "Amplitude der MEPs“ für die Messwiederholungsfaktoren "Zeitverlauf“, "tDCS-Bedingung" und Interaktion zwischen "Zeitverlauf $x$ tDCS-Bedingung" mit dazugehörigen Freiheitsgraden (d.f.), berechnetem F-Wert und $p$-Wert. Die Sternchenmarkierung * bedeutet einen als signifikant zu bewertenden p-Wert von $\leq$ 0,05 . Es zeigen sich signifikante Effekte für den Faktor „tDCS-Bedingung" und die Interaktion „Zeitverlauf $x$ tDCS-Bedingung“.

\begin{tabular}{|l|l|l|l|l|}
\hline & Variablen & $\begin{array}{l}\text { Freiheitsgrade } \\
\text { (d.f.) }\end{array}$ & F-Wert & P-Wert \\
\hline Experiment 1 & Zeitverlauf & 11 & 1,392 & 0,182 \\
\hline & tDCS-Bedingung & 5 & 4,239 & $0,002^{*}$ \\
\hline & $\begin{array}{l}\text { Zeitverlauf X tDCS- } \\
\text { Bedingung }\end{array}$ & 11 & 1,638 & $0,003^{*}$ \\
\hline
\end{tabular}

Die Post-Hoc-Tests zeigten, dass eine anodale tDCS von 13 Minuten eine Steigerung der neuronalen Erregbarkeit des motorischen Kortex relativ zur MEP- 
Amplitude vor tDCS zur Folge hatte, welche von der 5. bis zur 60. Minute nach Stimulation anhielt. Weiterhin bewirkte eine kontinuierliche anodale tDCS von 26 Minuten eine kortikale Erregbarkeitsverminderung relativ zur Ausgangsmessung vor tDCS und auch gegenüber der 13-minütigen kontinuierlichen anodalen tDCS. Dies war für 120 Minuten nachweisbar (siehe Abbildung 1).

\section{Abbildung 1: Kontinuierliche anodale tDCS (13/26 min)}

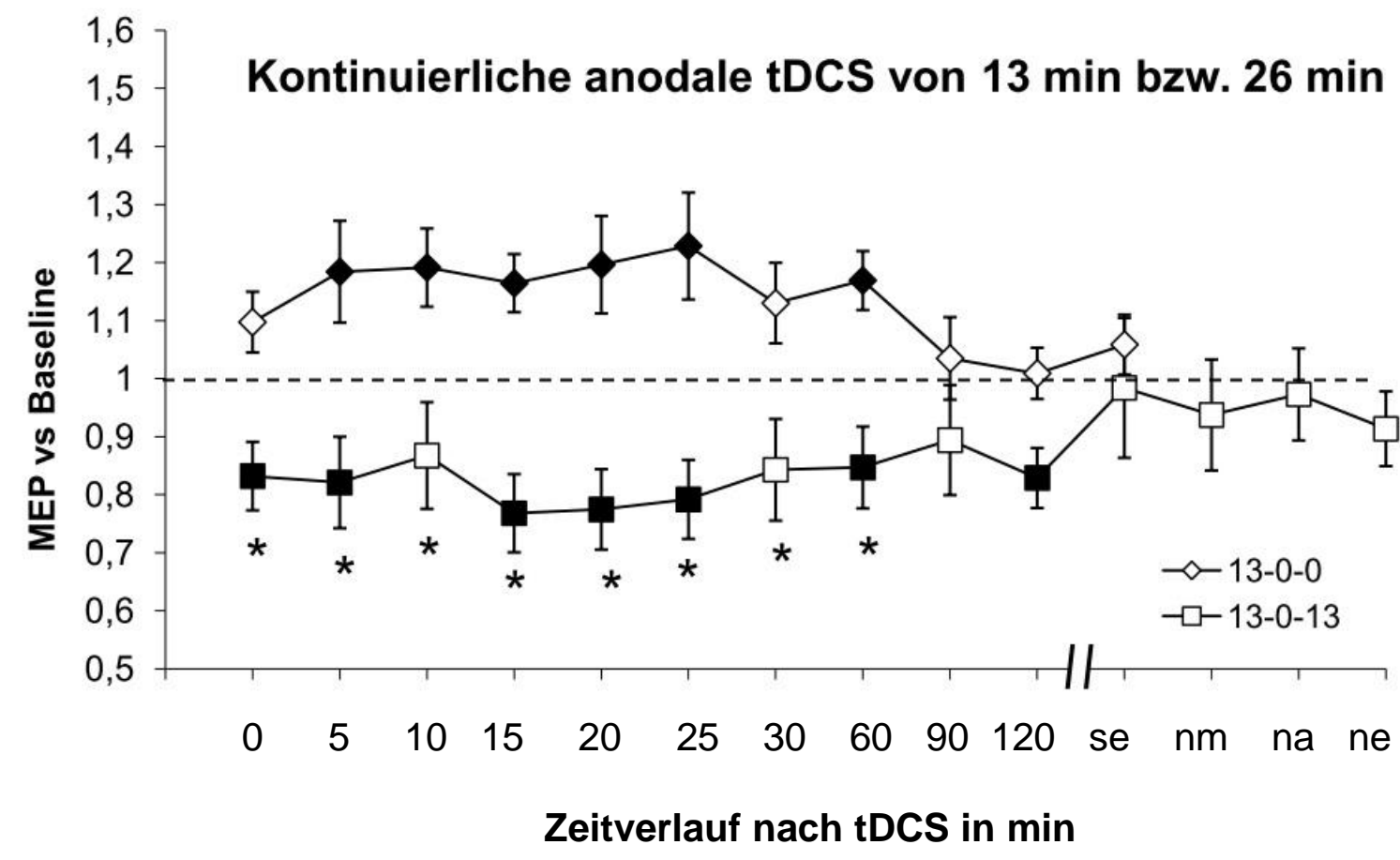

Für jeden Messzeitpunkt nach erfolgter tDCS sind die dazugehörigen Werte der MEPs eingetragen. Ausgefüllte Symbole bedeuten eine signifikante Abweichung von den MEPs der Ausgangsmessung vor tDCS. Eine Sternchenmarkierung * unter den Symbolen bedeutet für diesen Messzeitpunkt eine signifikante Veränderung im Vergleich zur kontinuierlichen anodalen tDCS von 13 Minuten. Die Fehlerbalken stellen den Standardfehler dar.

Diese Abbildung zeigt die Auswirkungen kontinuierlicher anodaler Gleichstromstimulation von 13 Minuten (Rautensymbol) und 26 Minuten (Quadratsymbol) auf die MEP-Amplitudenhöhe.

Für jeden Messzeitpunkt nach tDCS bis zur 60. Minute weicht die MEPAmplitudenhöhe zwischen den beiden Stimulationsformen signifikant voneinander ab (symbolisiert durch *). Eine kontinuierliche 13-minütige anodale tDCS führt zu einer Steigerung der kortikalen Erregbarkeit bis zur 60. Minute, wohingegen eine 26minütige anodale tDCS eine kortikale Erregbarkeitsverminderung bis zur 120. Minute zur Folge hat. 
Jene Stimulationsbedingungen mit wiederholter anodaler Gleichstromstimulation, die durch relativ kurze Pausen voneinander getrennt waren (3 Minuten, 20 Minuten), ließen initial einen Trend der gesteigerten kortikalen Erregbarkeit erkennen, der im Vergleich zur Baseline nicht signifikant war. Dieser Trend war für die Stimulationssitzung mit 3-minütiger Pause nach der 30. Minute und bei einer 20minütigen Pause nach der 90. Minute nicht mehr nachweisbar. Im Vergleich zu den Ergebnissen der kontinuierlichen 13-minütigen anodalen tDCS war diese frühe Erregbarkeitssteigerung des motorischen Kortex, welche sich in der Höhe der MEPAmplitude widerspiegelte, nur geringfügig niedriger. Schließlich war ein signifikanter Anstieg der neuronalen Erregbarkeit relativ zur Ausgangsmessung vor tDCS und zur 13-minütigen anodalen tDCS am Abend des Stimulationstages zu verzeichnen. Dieser Effekt war noch bis zum Abend des nächsten Tages und somit 24 Stunden nach Stimulation nachweisbar. Die kortikale Erregbarkeitssteigerung am Folgetag war für die Stimulationsbedingung mit einem stimulationsfreien Intervall von 20 Minuten am stärksten ausgeprägt (siehe Abbildung 2).

\section{Abbildung 2: Repetitive anodale tDCS mit kurzen Pausen}

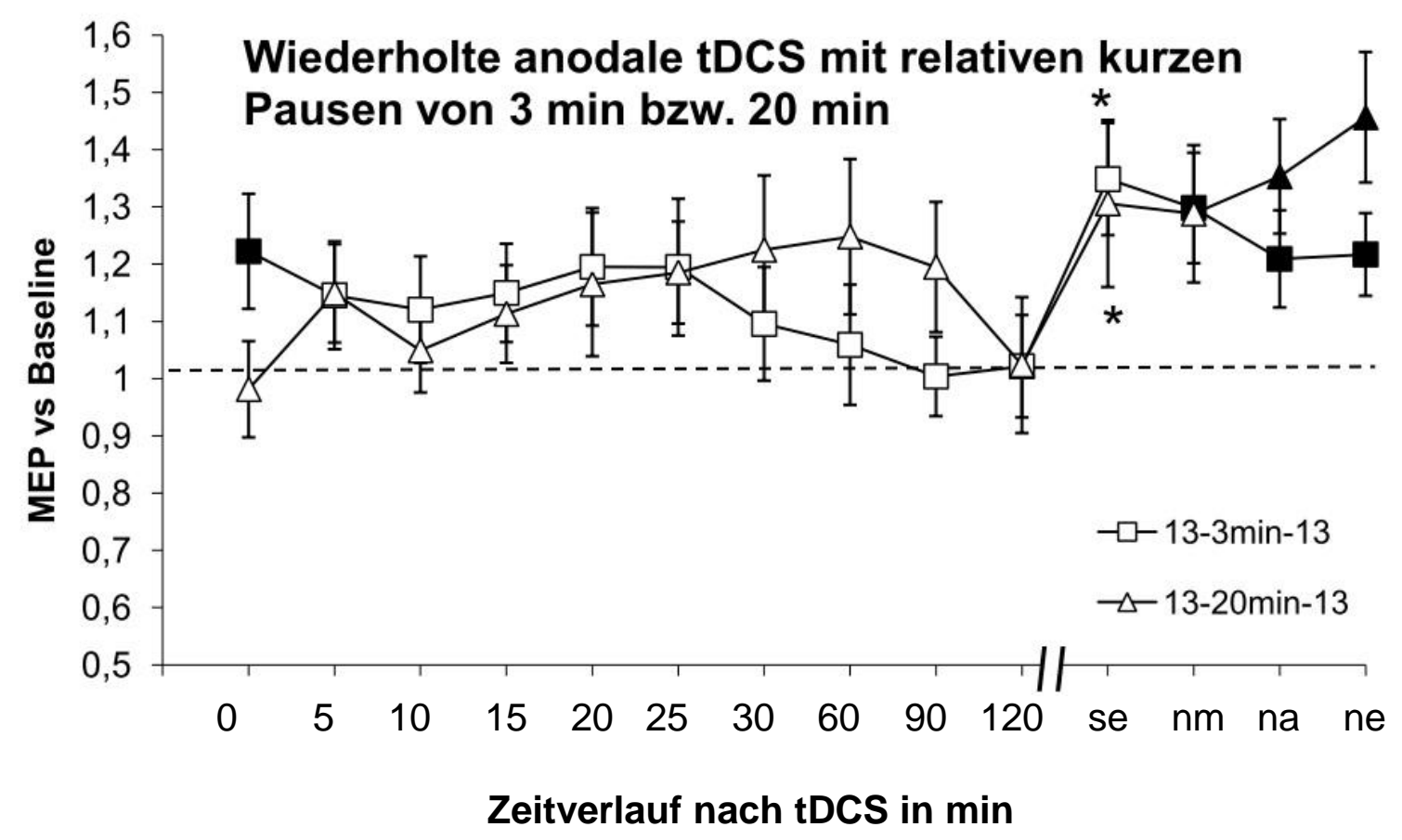

Für jeden Messzeitpunkt nach erfolgter tDCS sind die dazugehörigen Werte der MEPs eingetragen. Ausgefüllte Symbole bedeuten eine signifikante Abweichung von den MEPs der Ausgangsmessung vor tDCS. Die Sternchenmarkierung * unter den 
Symbolen bedeutet für diesen Messzeitpunkt eine signifikante Veränderung im Vergleich zur kontinuierlichen anodalen tDCS von 13 Minuten. Die Fehlerbalken stellen den Standardfehler dar. Diese Abbildung verdeutlicht die Effekte repetitiver anodaler Gleichstromstimulation mit relativ kurzen Pausen von 3 Minuten (Quadratsymbol) bzw. 20 Minuten (Dreiecksymbol) auf die MEP-Amplitudenhöhe. Unter beiden Bedingungen kommt es zu einem deutlichen Anstieg der Erregbarkeit des motorischen Kortex am Abend des Stimulationstages und am nachfolgenden Tag.

Jene Sitzungen, bei denen eine relativ lange Pause zwischen den repetitiven Stimulationen lag (3 Stunden oder 24 Stunden), zeigten keine eindeutigen Effekte hinsichtlich einer kortikalen Erregbarkeitsveränderung im Vergleich zur Ausgangsmessung der MEPs vor Stimulation. Allerdings war eine Tendenz der Erregbarkeitsverminderung im Vergleich zur Baseline einige Minuten nach der zweiten Stimulation $z u$ erkennen. Am Folgetag war eine diskrete Erregbarkeitssteigerung zu verzeichnen. Im Vergleich zur kontinuierlichen 13minütigen anodalen tDCS wichen beide Stimulationsbedingungen zu mehreren Zeitpunkten bis zur 60. Minute signifikant voneinander ab (symbolisiert durch *) und ließen dadurch erregbarkeitsvermindernde Effekte erkennen (siehe Abbildung 3).

\section{Abbildung 3: Repetitive anodale tDCS mit langen Pausen}

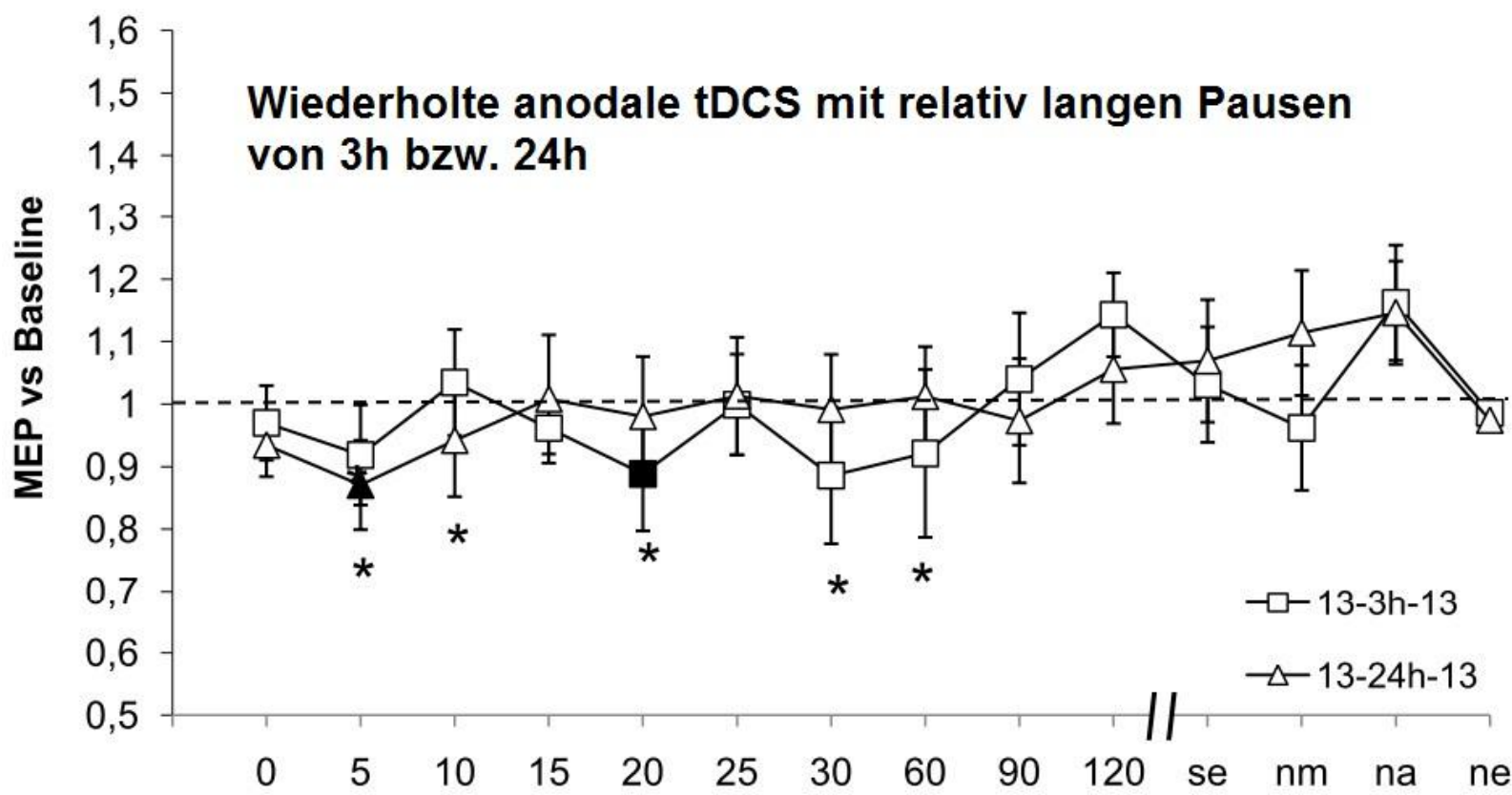

Zeitverlauf nach tDCS in min

Für jeden Messzeitpunkt nach erfolgter tDCS sind die dazugehörigen Werte der MEPs eingetragen. Ausgefüllte Symbole bedeuten eine signifikante Abweichung von 
den MEPs der Ausgangsmesssung vor tDCS. Die Sternchenmarkierung * unter den Symbolen bedeutet für diesen Messzeitpunkt eine signifikante Veränderung im Vergleich zur kontinuierlichen anodalen tDCS von 13 Minuten. Die Fehlerbalken stellen den Standardfehler dar. Die Abbildung 3 zeigt die Veränderung der Amplituden der MEPs im zeitlichen Verlauf für die Messungen der repetitiven anodalen Gleichstromstimulation mit relativ langen Pausen von 3 Stunden (Quadratsymbol) und 24 Stunden (Dreiecksymbol). Lediglich 2 einzelne Messpunkte (gefüllte schwarze Symbole) heben sich deutlich von der Baseline ab. Im Vergleich zur kontinuierlichen 13-minütigen anodalen Gleichstromstimulation ist das kortikale Aktivitätslevel deutlich gesunken.

Die Ergebnisse des Experiments 1 sprechen dafür, dass die Effekte repetitiver anodaler tDCS auf die Erregbarkeit des motorischen Kortex von der Dauer des stimulationsfreien Intervalls bestimmt werden.

\subsection{Ergebnisse des Experiments 2}

In diesem Experiment wurden die ursächlichen Mechanismen des Ergebnisses kontinuierlicher 26-minütiger anodaler tDCS an einer Subgruppe von 5 Probanden des 1. Experiments mittels pharmakologischer Intervention durch $10 \mathrm{mg}$ des Kalziumkanalblockers Flunarizin untersucht.

Das Niveau der MEP-Amplituden der Ausgangsmessung vor tDCS wurde durch Flunarizin nicht nachhaltig beeinflusst (Ausgangsmessung 1 vor Medikation: 1,002 $\mathrm{mV}+/-0,143 \mathrm{sd}$; Ausgangsmessung 2 nach Medikamenteneinnahme: 1,008 mV +/$0,105 \mathrm{sd}$; Studentscher $\mathrm{t}$-Test $\mathrm{p}=0,913$ ), weiterhin differierten die Baselines mit oder ohne medikamentösen Einfluss nicht untereinander (Ausgangsmessung 3 mit Flunarizin: 1,008 mV +/- 0,105; Ausgangsmessung 4 ohne Flunarizin: 1,092 mV +/0,058 ; Studentscher t-Test $p=0,233$ ).

Die ANOVA ergab einen signifikanten Haupteffekt des Faktors "Medikation“ (F-Wert: 30,634; Df-Wert: 1; p-Wert=0,005) (siehe Tabelle 3). 
Tabelle 3: Ergebnisse der ANOVA des Experiments 2

Daten der Messwiederholungs-ANOVA des Experiments 1 mit der abhängigen Variable „Amplitude der MEPs“ für die Messwiederholungsfaktoren "Zeitverlauf“, "Medikation" und Interaktion zwischen "Zeitverlauf x Medikation" mit dazugehörigen Freiheitsgraden (d.f.= degrees of freedom), berechnetem F-Wert und $p$-Wert.

Die Sternchenmarkierung * bedeutet einen als signifikant zu bewertenden $p$-Wert von $\leq 0,05$.

\begin{tabular}{|l|l|l|l|l|}
\hline & Variablen & $\begin{array}{l}\text { Freiheitsgrade } \\
\text { (d.f.) }\end{array}$ & F-Wert & P-Wert \\
\hline Experiment 2 & Zeitverlauf & 10 & 1,704 & 0,114 \\
\hline & Medikation & 1 & 30,634 & $0,005^{\star}$ \\
\hline & Zeitverlauf x Medikation & 10 & 1,176 & 0,335 \\
\hline
\end{tabular}

Die Auswertung der Post-Hoc-Tests zeigte, dass durch den Einsatz des Kalziumkanalblockers Flunarizin die inhibitorische Wirkung der kontinuierlichen 26minütigen anodalen tDCS auf die kortikale Erregbarkeit aufgehoben wurde (siehe Abbildung 4)

Abbildung 4: Kontinuierliche anodale tDCS (26 min)+/- Medikation



Zeitverlauf nach tDCS in min

Für jeden Messzeitpunkt nach erfolgter tDCS sind die dazugehörigen Werte der MEPs eingetragen. Ausgefüllte Symbole bedeuten eine signifikante Abweichung von 
den MEPs der Ausgangsmessung vor tDCS. Die Sternchenmarkierung * unter den Symbolen bedeutet für diesen Messzeitpunkt eine signifikante Veränderung im Vergleich zur kontinuierlichen anodalen tDCS von 13 Minuten. Die Fehlerbalken stellen den Standardfehler dar.

Runde Symbole stehen für die Stimulationsbedingung 26-minütiger anodaler tDCS mit vorheriger Einnahme von $10 \mathrm{mg}$ des Kalziumkanalblockers Flunarizin. Quadratische Symbole entsprechen einer einfachen kontinuierlichen 26-minütigen anodalen tDCS ohne Medikation.

Während die Messwerte der 26-minütigen kontinuierlichen anodalen Gleichstromstimulation signifikant von der Baseline abweichen und deren inhibitorischen Effekt anzeigen, bewegen sich die Werte der MEPs bei zusätzlicher Medikation von Flunarizin im Bereich der Baseline. Innerhalb der ersten 30 Minuten nach $t D C S$ unterscheiden sich die MEP-Amplituden in den Bedingungen mit und ohne Flunarizin signifikant voneinander (symbolisiert durch *).

\subsection{Zusammenfassung des Ergebnisteils}

Zusammenfassend kommt die Studie zu folgenden Ergebnissen:

Eine kontinuierliche anodale Gleichstromstimulation von 13 Minuten lieferte eine Erregbarkeitssteigerung des motorischen Kortex, die bis zu 60 Minuten nach Stimulation nachweisbar war. Eine Verdopplung der anodalen Stimulationsdauer führte in Gegenüberstellung zur MEP-Ausgangsmessung vor tDCS und zur kontinuierlichen anodalen 13-minütigen tDCS zu einer Erregbarkeitsverminderung, die sich ebenfalls über eine Stunde nachweisen ließ. Eine repetitive 13-minütige anodale Gleichstromstimulation mit relativ kurzen Pausen zeigte gegenüber der Ausgangsmessung vor tDCS während des Stimulationstages eine Tendenz zur Erregbarkeitssteigerung, welche im Vergleich zur kontinuierlichen 13-minütigen anodalen tDCS nur geringfügig abgefallen war. Darüber hinaus mündete dieser Trend am Abend des Stimulationstages in einer signifikanten Erhöhung der Erregbarkeit - sowohl hinsichtlich der Ausgangsmessung vor tDCS als auch hinsichtlich der kontinuierlichen 13-minütigen anodalen tDCS. Diese kortikale Erregbarkeitssteigerung konnte bis zur abendlichen Messung am Folgetag und somit 24 Stunden nach Stimulation beobachtet werden. Repetitive 13-minütige anodale Gleichstromstimulation mit langen Pausen ließ im Vergleich zur Ausgangsmessung vor tDCS während des Stimulationstages nur ansatzweise inhibitorische und am darauffolgenden Tag exzitatorische Effekte erkennen. Verglichen mit kontinuierlicher 13-minütiger anodaler tDCS war eine deutliche Verminderung der Erregbarkeit zu verzeichnen. Das Experiment 2 zeigte, dass die erregbarkeitsvermindernden Effekte 
der kontinuierlichen 26-minütigen anodalen Gleichstromstimulation durch den Einsatz des Kalziumkanalblockers Flunarizin verhindert wurden.

\subsection{Verträglichkeit der tDCS}

Insgesamt wurden sowohl die kontinuierliche als auch die repetitive anodale tDCS gut vertragen. Während der anodalen Gleichstromstimulation bemerkten die Probanden ein leichtes Jucken unter den Elektroden, welches durch erneutes Befeuchten der Schwammelektroden mit 0,9\% NaCl-Lösung schnell gebessert werden konnte. Zusätzlich traten leichte Rötungen der Kopfhaut an den Stimulationsstellen auf, welche nicht schmerzhaft waren und innerhalb eines Zeitraums von ca. 30 Minuten wieder verschwanden. Weitere sporadisch auftretende Nebenwirkungen waren Müdigkeit und leichte Kopfschmerzen. Die beschriebenen unerwünschten Wirkungen blieben durch die Einnahme des Kalziumkanalblockers Flunarizin unverändert.

Keiner der Probanden beendete vorzeitig seine Studienteilnahme aufgrund der beschriebenen Nebenwirkungen. 


\section{Diskussion}

\section{1 Überblick}

Diese Arbeit untersuchte die Effekte von repetitiver anodaler Gleichstromstimulation auf die neuronale Erregbarkeit des motorischen Kortex. Hierbei konnte gezeigt werden, dass eine wiederholte anodale Gleichstromstimulation in der Lage ist, langanhaltende Erregbarkeitssteigerungen $\mathrm{zu}$ induzieren, welche mehr als 24 Stunden nach Stimulation nachweisbar waren. Dies könnte durch die Etablierung von synaptischer Effizienz im Sinne des Phänomens der Langzeitpotenzierung respektive der Spätphase der Langzeitpotenzierung (I-LTP) erklärt werden. Von entscheidender Bedeutung für die Induktion einer solchen neuronalen Plastizitätsveränderung waren der zeitliche Verlauf der Stimulation und vor allem die Dauer des freien Intervalls zwischen zwei Stimulationen. Wurde dieses so gewählt, dass die zweite Stimulation während der Nacheffekte der ersten einsetzte - wie bei den beiden Sitzungen mit relativ kurzen Pausen von 3 Minuten oder 20 Minuten - so verlängerten sich die exzitatorischen Effekte der Stimulation bis zum Abend des darauffolgenden Tages. Ganz anders fielen die Resultate bei einem länger gewählten stimulationsfreien Intervall von 3 Stunden oder 24 Stunden aus. Dabei setzte die zweite Stimulation zu einem Zeitpunkt ein, an dem die Effekte der ersten Stimulation bereits beendet waren, und führte zu keinem signifikanten Erregbarkeitsanstieg. Eine mögliche Erklärung dafür könnte das Auftreten homöostatischer Mechanismen sein, die einer ungehemmten, überschießenden Erregung vorbeugen, um die Stabilität des neuronalen Verbundes zu gewährleisten.

Der zeitliche Abstand zwischen den repetitiven Stimulationen war der maßgebliche Faktor zur Induktion von langanhaltenden Nacheffekten auf die kortikale Erregbarkeit. Denn eine kontinuierliche anodale Gleichstromstimulation der gleichen Gesamtstimulationsdauer wie die repetitiven Stimulationen führte zu einem vollkommen anderen Effekt: einer Erregbarkeitsverminderung bis zur 60. Minute nach Stimulationsende.

Im Folgenden werde ich zunächst die Stimulationsbedingungen des ersten Experiments anhand der aktuellen Studienlage diskutieren. Zu Beginn beschreibe ich kurz die Effekte kontinuierlicher 13-minütiger anodaler tDCS. Diese Stimulationsform 
diente in dieser Arbeit als Kontrollbedingung und wurde bereits in anderen Studien ausführlich diskutiert. Anschließend werde ich die Sitzungen repetitiver anodaler tDCS analysieren und schließlich die Hintergrundmechanismen der Effekte 26minütiger anodaler tDCS über dem humanen Motorkortex des ersten und zweiten Experiments erörtern.

Am Ende der Diskussion werde ich eine Zusammenfassung liefern, die die funktionellen Aspekte der Ergebnisse, die möglichen klinischen Anwendungsgebiete und die Limitierungen dieser Studie beinhalten wird.

\subsection{Kontinuierliche 13-minütige anodale tDCS}

Induktion einer langanhaltenden kortikalen Exzitabilitätssteigerung bis zu einer Stunde nach Stimulationsende

Die Auswirkungen kontinuierlicher 13-minütiger anodaler Gleichstromstimulation auf die Erregbarkeit und Aktivität des motorischen Kortex wurden bereits in früheren Studien untersucht (Nitsche und Paulus 2001; Nitsche et al. 2003a, 2004a). Die Stimulationsform diente in diesem Experiment lediglich als Kontrollbedingung, um herauszufinden, ob länger anhaltende Nacheffekte durch die Applikation von repetitiven Stimulationen erzeugt werden können. Die Ergebnisse dieser Arbeit stehen im Einklang mit früheren Ergebnissen und bestätigen einen exzitabilitätssteigernden Effekt bis zur 60. Minute nach Stimulationsende. Zwar sind die MEP-Amplituden etwas geringer als im Vergleich zur o.g. Studie von 2001 und auch die Dauer der Nacheffekte ist etwas kürzer, doch dies könnte durch das unterschiedliche Probandenkollektiv bedingt sein.

Als ursächliche Mechanismen der bis zu einer Stunde nach Stimulation nachweisbaren Exzitabilitätssteigerung wurden eine erhöhte NMDA-Rezeptoraktivität und eine erhöhte Konzentration von intrazellulärem Kalzium beschrieben, die damit einer Plastizitätsveränderung im Sinne der Langzeitpotenzierung (LTP) ähneln (Malenka und Bear 2004; Nitsche und Paulus 2001; Nitsche et al. 2003a, 2004a). 


\subsection{Repetitive anodale tDCS mit kurzen Pausen}

Auslösung I-LTP-ähnlicher Plastizität durch repetitive anodale Gleichstromstimulation mit einem stimulationsfreien Intervall von 3 Minuten bzw. 20 Minuten

Wurde die repetitive anodale Gleichstromstimulation durch eine Pause von 3 Minuten oder 20 Minuten voneinander separiert, so zeigte sich nach Stimulationsende ein zweiphasiger Verlauf der MEP-Amplitude. Zunächst war ein Trend zur Steigerung der Erregbarkeit im Vergleich zur Ausgangsmessung vor tDCS bis zur 90. Minute nach anodaler Gleichstromstimulation erkennbar. Danach folgte ein Abfall der MEPAmplitude auf das Niveau der Baseline nach 2 Stunden. Schließlich war am Abend des Stimulationstages und am darauffolgenden Tag eine deutliche Erregbarkeitssteigerung im Vergleich zur Baseline zu verzeichnen.

Die ansatzweise erkennbare Steigerung der neuronalen Aktivität des motorischen Kortex direkt nach Stimulationsende bis zur 90. Minute nach tDCS ist vergleichbar mit der Erregbarkeitssteigerung der einfachen kontinuierlichen 13-minütigen anodalen Gleichstromstimulation, auch wenn diese etwas geringer ausfällt. Aufgrund dieser Parallelen scheint es naheliegend, die bereits oben erwähnten Faktoren der erhöhten NMDA-Rezeptor-Aktivität und die erhöhte intrazelluläre Kalziumkonzentration als Ursache zu vermuten. Eben diese beiden Faktoren sind auch für die Induktion der Langzeitpotenzierung verantwortlich (Malenka und Bear 2004). Diese Form von synaptischer Plastizität konnte erstmals 1973 im Tierversuch mittels hochfrequenter, tetanischer Stimulation des Hippokampus durch Bliss und Lomo nachgewiesen werden. Die Langzeitpotenzierung ist durch eine gesteigerte Effizienz synaptischer Verbindungen gekennzeichnet, die länger als 30 Minuten nach Stimulation nachweisbar ist. Wegen der beschriebenen Gemeinsamkeiten werden die durch tDCS verursachten langanhaltenden kortikalen Erregbarkeitsveränderungen als LTP-ähnliche Plastizität bezeichnet (Nitsche und Paulus 2000, 2001). Auch andere nicht-invasive Stimulationsformen haben humanexperimentell eine LTP-ähnliche Plastizität bewirken können (Huang $\mathrm{YZ}$ et al. 2007; Stefan et al. 2000, 2002; Ziemann und Siebner 2008). Diese Plastizitätsform lässt sich in eine frühe und eine späte Phase aufteilen, die sich in ihrem zeitlichen Auftreten und in den auf molekularer Ebene ablaufenden Prozessen voneinander unterscheiden. Analog dazu könnte die nach Stimulationsende nachweisbare Exzitabilitätserhöhung einer Langzeitpotenzierung der Frühphase entsprechen (e- 
LTP). Dies ist eine synaptische Plastizitätsveränderung, welche ca. 1-3 Stunden anhalten kann (Frey et al. 1993) und aus molekularer Sicht durch folgende Abläufe geprägt ist: ein über NMDA-Rezeptoren vermittelter erhöhter $\mathrm{Ca}^{2+}$-Einstrom in die Nervenzelle aktiviert Kalzium-Calmodulin-Kinasen, die über weitere Signalkaskaden dafür sorgen, dass AMPA-Rezeptoren, die die Untereinheit Glu-R1 enthalten, vermehrt in die postsynaptische Membran eingebaut werden (Feldman 2009; Malenka und Bear 2004; Malinow und Malenka 2002). Dies führt über den Neurotransmitter Glutamat zu erhöhten exzitatorischen Potentialen.

Die Abnahme der Erregbarkeit zum Zeitpunkt zwei Stunden nach Ende der Stimulation könnte durch kurzfristige Homöostaseeffekte verursacht sein. Diesen Mechanismus werde ich im weiteren Verlauf der Diskussion noch näher erläutern.

Hervorzuheben ist an dieser Stelle der signifikante Anstieg der neuronalen Aktivität des motorischen Kortex drei Stunden nach Ende der Stimulation bis in den Abend des Folgetages hinein. Dieses Ergebnis teilt einige Gemeinsamkeiten mit dem Phänomen der Langzeitpotenzierung der Spätphase (I-LTP), welches bereits in Tierversuchen an Neuronen der CA1-Region des Hippokampus untersucht wurde (Frey et al. 1993). Repetitive Stimulationen mit dazwischenliegenden Pausen von 510 Minuten führten zu einer Erregbarkeitssteigerung, die mehr als drei Stunden nach Stimulation nachweisbar war und für eine Dauer von mehreren Stunden in tierischen Hippokampus-Präparaten oder aber mehrere Tage in vivo anhielt (Huang YY et al. 1996, 2004). Auch Gong et al. konnten 2011 in den retinotektalen Synapsen von Xenopus laevis (glatter Krallenfrosch) zeigen, dass repetitive Theta-Burst-Stimulation mit stimulationsfreien Intervallen von 5 Minuten zur Induktion von I-LTP in der Lage ist, wohingegen dieselbe Stimulationsdauer ohne dazwischenliegende Pausen lediglich zu e-LTP führte. Des Weiteren konnten auch Trepel und Racine 1998 eine LTP-ähnliche Plastizität im Neokortex einer Ratte in vivo induzieren, sofern es sich um wiederholte, voneinander separierte Stimulationen an mindestens 10 aufeinanderfolgenden Tagen handelte.

Zur Ausbildung eines I-LTP im Tierexperiment sind Proteinkinase-A-Aktivität, Proteinund RNA-Synthese vonnöten (Huang YY et al. 1996). Der Einsatz des Proteinsyntheseinhibitors Anisomycin verhinderte vollständig das Auftreten eines ILTP in vitro (Frey et al. 1988). Weitere Faktoren innerhalb der Signalkaskade zur Induktion eines I-LTP sind die MAP-Kinase und der Transkriptionsfaktor CERB (Malenka und Bear 2004). Tier- und humanexperimentell lassen sich folgende 
Gemeinsamkeiten für die Induktion von LTP-ähnlicher Plastizität zusammenfassen: Zur Auslösung dieser Form der synaptischen Plastizität wird im Tier- (Cooke und Bliss 2006; Malenka und Bear 2004) und im Humanexperiment (Huang YY et al. 2007; Nitsche et al. 2003b; Stefan et al. 2000, 2002) NMDA-Rezeptoraktivität benötigt. Im Tierversuch wurde in vitro und in vivo die Ausbildung von LTP durch die Anwendung eines NMDA-Rezeptorantagonisten wie z.B. AP5 und Dextromethorphan verhindert (Collingridge et al. 1983; Krug et al. 1993). In gleicher Weise blockierten die NMDA-Antagonisten Dextromethorphan (Nitsche et al. 2003a; Stefan et al. 2000) oder Memantine (Huang YY et al. 2007) die Induktion LTP-ähnlicher Plastizität in verschiedenen Stimulationsbedingungen im Menschen. Weiterhin konnte eine länger als drei Stunden anhaltende neuronale Erregbarkeitssteigerung im Tierexperiment nur durch repetitive Stimulationsformen erreicht werden - wie oben mehrfach beschrieben - und auch die tDCS konnte dies erstmalig in dieser Studie im Humanexperiment durch eine repetitive anodale Stimulationsform zeigen. Außerdem gleichen sich die Studien an Tieren und die Ergebnisse dieses Experiments in Bezug auf die späte Phase der Langzeitpotenzierung darin, dass die Pausen zwischen den Stimulationen relativ kurz waren: im Tierexperiment wird ein stimulationsfreies Intervall von 5-10 Minuten zwischen hochfrequenten tetanischen Stimulationen beschrieben (Frey et al. 1993; Gong et al. 2011; Huang YY et al. 1996) und in dieser Arbeit waren Pausen von 3 Minuten oder 20 Minuten effektiv zur Auslösung I-LTPähnlicher Plastizität.

Diese Vielzahl von Parallelen zwischen Tierversuchen und den Ergebnissen dieser Arbeit spricht dafür, dass man erstmalig von der Erzeugung I-LTP-ähnlicher synaptischer Plastizität im Menschen sprechen kann.

Allerdings bleiben die Erklärungen dafür auf molekularer Ebene Spekulation. Die genauen Mechanismen der durch tDCS ausgelösten Erregbarkeitsveränderungen des motorischen Kortex dieser Studie sind noch nicht bekannt. Möglicherweise ist wie im Tierexperiment die Synthese neuer Proteine der ausschlaggebende Faktor doch diese Vermutung werden nachfolgende Studien untersuchen müssen. 


\title{
4.4 Repetitive anodale tDCS mit langen Pausen
}

\author{
Erzeugung von homöostatischer Plastizität durch repetitive anodale \\ Gleichstromstimulation mit einem stimulationsfreien Intervall von mehreren Stunden
}

Die Ergebnisse der repetitiven anodalen Gleichstromstimulation mit relativ langen Pausen von 3 Stunden oder 24 Stunden differierten erheblich von den Resultaten der repetitiven Stimulationen mit einem stimulationsfreien Intervall von wenigen Minuten und konnten auch die von mir zu Beginn der Arbeit formulierte Hypothese nicht bestätigen. In dieser Stimulationsbedingung erfolgte die zweite Stimulation zu einem Zeitpunkt, zu dem die Nacheffekte der ersten Stimulation bereits beendet waren. Nichtsdestotrotz kam es im Anschluss weder zu einer Erregbarkeitssteigerung noch zu einem deutlichen Absinken der Erregbarkeit, vielmehr verharrte das Niveau der MEP-Amplitude während des gesamten Messzeitraums ungefähr auf dem Niveau der Ausgangsmessung vor tDCS. Verglichen mit der einfachen kontinuierlichen 13minütigen anodalen tDCS zeigten sich erregbarkeitsvermindernde Effekte während der 1. Stunde nach Stimulationsende. Die Induktion von homöostatischer Plastizität könnte ein möglicher Grund für das Ausbleiben einer Erregbarkeitssteigerung der Neurone des motorischen Kortex sein. Homöostatische Plastizität beschreibt das Phänomen einer negativen Rückkopplungskontrolle, bei der ein eingehender Stimulus innerhalb eines Neurons, einer Synapse oder eines übergeordneten Netzwerkes von Neuronen eine Plastizitätsveränderung in Abhängigkeit von dem vorherrschendem Aktivitätsniveau bewirkt, sodass eine überschießende Fazilitierung oder Inhibition vermieden wird, um eine adäquate Funktionsfähigkeit und Stabilität der oben genannten Funktionseinheiten zu gewährleisten (Turrigiano 2008; Zhao et al. 2011). Als erstes wurde das Modell der "homöostatischen Plastizität" in der Bienenstock-Cooper-Munro-Regel 1982 (BCM) theoretisch beschrieben. Diese besagt, dass die Art der Erregbarkeitsveränderung eines Neurons von der vorherrschenden Aktivität der postsynaptischen Membran abhängig ist. Weiterhin wird eine Reizschwelle beschrieben, welche zur Induktion von LTP-ähnlicher Plastizität überschritten werden muss. Im Fall eines erhöhten Aktivitätsniveaus der postsynaptischen Membran steigt diese an, wodurch die Wahrscheinlichkeit der Ausbildung von LTP geringer wird. Bei einem erniedrigten Aktivitätsniveau der postsynaptischen Membran sinkt die Reizschwelle und erleichtert damit die Induktion 
eines LTP (Bienenstock et al. 1982; Siebner und Ziemann 2007). Diese Theorie konnte in zahlreichen Tierversuchen in den letzten Jahren bestätigt werden (Abraham et al. 2001; Desai et al. 2002; Huang YY et al. 1992; Kirkwood et al. 1996). Das Auftreten von homöostatischer Plastizität im menschlichen Kortex konnte bereits in vielen Studien durch die Anwendung nicht-invasiver Stimulationsverfahren nachgewiesen werden (Lang et al. 2004; Müller et al. 2007; Nitsche et al. 2007; Ziemann et al. 2008).

In einer Studie von 2003 demonstrierten lyer et al. dies erstmalig mittels Kombination von niederfrequenter und hochfrequenter transkranieller Magnetstimulation. Im Anschluss daran konnte gezeigt werden, dass Homöostaseeffekte innerhalb des motorischen Kortex auch durch die Kombination von tDCS und rTMS induziert werden können (Lang et al. 2004; Siebner et al. 2004): Wurde eine erregbarkeitssteigernde oder -reduzierende transkranielle Gleichstromstimulation von einer gleichgerichteten transkraniellen Magnetstimulation gefolgt, so führte dies im Ergebnis zu einer entgegengesetzten Änderung des Aktivitäts- und Erregbarkeitsniveaus. Erst kürzlich wurde damit begonnen zu untersuchen, ob auch repetitive transkranielle Gleichstromstimulation zur Induktion von Homöostaseeffekten führt. Monte-Silva et al. konnten 2010 in einer Studie zu repetitiver kathodaler tDCS übereinstimmend mit den in dieser Studie präsentierten Ergebnissen zeigen, dass ein stimulationsfreies Intervall von 3 Stunden oder 24 Stunden zu homöostatischer Plastizität führt. Darüber hinaus konnte eine weitere Studie zu repetitiver transkranieller Gleichstromstimulation mit sehr viel kürzeren Stimulationszeiten und dazwischenliegenden Pausen von nur wenigen Minuten ebenfalls Effekte erzeugen, die mit homöostatischen Mechanismen vereinbar sind (Fricke et al. 2011). Da in diesem Fall schon nach wenigen Minuten homöostatische Plastizität induziert werden konnte, müssen andere zelluläre und molekulare Mechanismen vermutet werden, als bei der Form, die in dieser Arbeit beschrieben wurde. Ein umfassendes Resumée der bislang bekannten verschiedenen zellulären und molekularen Mechanismen, von denen vermutet wird, dass sie zu homöostatischer Plastizität innerhalb des menschlichen Gehirns führen, erhält man durch die Übersichtsarbeit von Pozo und Goda aus 2010. Auf der zellulären Ebene scheint die Anpassung der Neurotransmitterausschüttung aus den Vesikeln der Präsynapse, der Einbau von Rezeptoren an der postsynaptischen Membran und die Veränderung der AMPA-Rezeptorendichte an der postsynaptischen Membran, 
welche Ausdruck der synaptischen Skalierung ist, eine entscheidende Rolle zu spielen. Der Begriff der synaptischen Skalierung beschreibt eine Form von homöostatischer Plastizität, bei der innerhalb eines neuronalen Netzwerkes die Stärke der synaptischen Übertragung aller Synapsen eines Neurons so angepasst wird, dass die relative Anzahl der Übertragungsimpulse beibehalten wird. Auf molekularer Ebene werden Transkriptionsprozesse und Proteinsynthese beschrieben, die über Signalmoleküle wie BDNF und TNFa moduliert werden.

Doch welcher dieser Mechanismen ist am ehesten ursächlich für die in diesem Experiment nachgewiesene homöostatische Plastizitätsveränderung?

$\mathrm{Da}$ tDCS bekanntermaßen über die Veränderung der intrazellulären Kalziumkonzentration und Effektivität von glutamatergen NMDA-Rezeptoren neuronale Plastizitätsveränderungen bewirkt, scheint der folgende von Turrigiano 2008 beschriebene Mechanismus am wahrscheinlichsten kausal zu sein: Ein erhöhtes Aktivitätsniveau des Neurons durch LTP-ähnliche Plastizitätsveränderung führt zu einem Überschuss an intrazellulärem Kalzium, was durch zelleigene Kalziumsensoren detektiert wird und in der Folge zu einer erhöhten Aktivität der CaMKIV (Kalziumcalmodulinkinase) führt. Diese wiederum führt im Nukleus der Zelle zu einer Verminderung des "Skalierungsfaktors", wodurch weniger glutamaterge Rezeptoren der Gruppe AMPA in die postsynaptische Membran eingebaut werden und die Reizschwelle zur Auslösung neuer Aktionspotentiale erhöht wird. Ob auch andere Signalmoleküle wie beispielsweise BDNF und TNFa oder aber ganz andere molekulare Prozesse als die bisher beschriebenen bei der Ausbildung homöostatischer Plastizität durch repetitive tDCS involviert sind, kann zur Zeit nicht sicher belegt oder ausgeschlossen werden. Weiterführende Studien werden sich dieser Problematik annehmen müssen.

In dieser Studie konnte erstmals gezeigt werden, dass repetitive anodale tDCS mit stimulationsfreien Intervallen von mehreren Stunden zur Ausbildung von homöostatischen Effekten innerhalb des motorischen Kortex führt. Interessanterweise traten diese Effekte zu einem Zeitpunkt auf, an dem die durch tDCS hervorgerufenen Plastizitätsveränderungen längst verschwunden waren. Zukünftige Studien sollten untersuchen, ob dies in Zusammenhang mit intrakortikalen Erregbarkeitsveränderungen steht, welche nicht notwendigerweise von Modifikationen der kortikospinalen Erregbarkeit begleitet sein müssen (Boros et al. 2008; Nitsche et al. 2005). 
Es steht zu vermuten, dass das Thema neuronaler homöostatischer Plastizität auch in naher Zukunft die Forschung beschäftigen wird, da die Ergebnisse zum jetzigen Zeitpunkt sehr uneinheitlich sind. Sowohl Studien, deren stimulationsfreies Intervall nur wenige Minuten betrug, als auch Studien mit einer stimulationsfreien Phase von mehreren Stunden konnten Homöostaseeffekte erzeugen. Gründe für die heterogenen und teilweise entgegengesetzten Ergebnisse der Studien könnten zum einen die unterschiedlichen Stimulationsformen und Kombinationen zwischen PAS, tDCS und rTMS (mit voneinander abweichenden zellulären und molekularen Wirkmechanismen), zum anderen aber auch die inhomogenen Probandenkollektive sein. Weitere Studien sollten diese Komponenten systematisch untersuchen.

\subsection{Kontinuierliche 26-minütige anodale tDCS}

Umkehr der Nacheffekte kontinuierlicher anodaler transkranieller Gleichstromstimulation bei Verlängerung der Stimulationsdauer

Überraschenderweise führte eine Verlängerung der Stimulationsdauer anodaler tDCS nicht zu einer Verlängerung der Nacheffekte im Sinne einer Erregbarkeitssteigerung, sondern erzielte einen genau entgegengesetzten Effekt nämlich eine signifikante Verminderung der Erregbarkeit bis zur 60. Minute nach Stimulationsende. Der Vergleich zwischen kontinuierlicher und repetitiver Stimulationsform macht deutlich, dass die Länge des stimulationsfreien Intervalls und damit der Zeitpunkt, zu dem die zweite Stimulation auf die Membranpotentiale der bereits stimulierten Neurone trifft, den entscheidenden Einfluss auf die Art der Nacheffekte im Sinne einer eher gesteigerten, verminderten oder gleichbleibenden kortikalen Erregbarkeit hat, weil die gleiche Stimulationsdauer "en masse“ angewandt einen konträren Effekt zur aufgespaltenen Stimulationsform hat. Analog dazu kamen auch Philips und Carew in ihrer Veröffentlichung von 2009 mit dem bezeichnenden Titel "It's all about timing“ zu dem Ergebnis, dass für die Formierung von Langzeiterinnerungen in regelmäßigen Abständen vollzogene Lerneinheiten mit der gleichen Gesamtdauer besser geeignet sind als eine kontinuierlich an einem Stück durchgeführte Trainigssitzung. Seit einiger Zeit ist bekannt, dass anodale tDCS zur Steigerung der Erregbarkeit und des Aktivitätsniveaus an Neuronen des motorischen Kortex führt (Nitsche und Paulus 2000, 2001). Bisher resultierte eine Verlängerung 
der Stimulationsdauer anodaler tDCS stets auch in einer Verlängerung der exzitabilitätserhöhenden Nacheffekte. Nitsche und Paulus zeigten 2000, dass eine 5minütige anodale tDCS über dem motorischen Kortex zu einer Erregbarkeitssteigerung führte, die bis zur 5 . Minute nach tDCS nachweisbar war. Im darauffolgenden Jahr steigerten sie die Stimulationsdauer auf 13 Minuten und erhielten dadurch gleichgerichtete Nacheffekte, die 90 Minuten anhielten. Die Ergebnisse in unserer Studie sind Zeugnis dafür, dass eine Erhöhung der Stimulationsdauer nicht zwangsläufig zu einer Verlängerung der Nacheffekte führt, sondern entgegengesetzte, stabilisierende Mechanismen von der Zelle generiert werden, möglicherweise, um einen Zusammenbruch des Netzwerkes mit unkontrollierter Erregungsausbreitung zu vermeiden. Ähnlich präsentiert sich eine Studie zu Theta-Burst-Stimulation (TBS), bei der die Verdopplung der Stimulationsdauer im Vergleich zu den ansonsten üblichen Stimulationsformen zu einem entgegengesetzten Effekt auf die kortikale Erregbarkeit führte (Gamboa et al. 2010). Die in unserer Studie erzielten Ergebnisse der kontinuierlichen 26-minütigen anodalen Gleichstromstimulation könnten auf molekularer Ebene durch den spannungsabhängigen Kv2.1-Kalium-Kanal ausgelöst worden sein. Dieser ist für die Kontrolle eines stabilen Membranpotentials der Neurone zuständig. Innerhalb der Pyramidenbahnzellen der Ratte konnte in vivo gezeigt werden, dass ein erhöhtes neuronales Aktivitätsniveau verbunden mit hohem Kalziumeinstrom intrazellulär zu einer Dephosphorylierung des Kaliumkanals mit anschließender Hyperpolarisation führt (Misonou et al. 2004, 2008). Dies hat in der Summe eine Abnahme der kortikalen Erregbarkeit zur Folge. Dass die intrazelluläre Kalziumkonzentration in Zusammenhang mit unseren Ergebnissen stehen muss, wurde durch den Einsatz des Kalziumkanalblockers Flunarizin im 2. Experiment bewiesen, da dieser den inhibitorischen Effekt der 26-minütigen anodalen tDCS verschwinden ließ und sich das Niveau der Erregbarkeit auf Höhe der Ausgangsmessung vor tDCS einpendelte. Doch dies ist nicht die einzige mögliche Erklärung der nachgewiesenen Resultate und nachfolgende Studien werden dieses Phänomen noch genauer untersuchen müssen. 


\subsection{Ausblick}

Diese Studie konnte unter Einsatz repetitiver anodaler Gleichstromstimulation erstmals den Nachweis von I-LTP-ähnlicher Plastizität im humanen Motorkortex erbringen. Für die Auslösung dieser Form von Neuroplastizität war die Dauer des stimulationsfreien Intervalls von maßgeblicher Bedeutung. Eine relativ kurze Pause zwischen den 13-minütigen Stimulationen, bei der die zweite Stimulation auf die Nacheffekte der ersten traf, führte zu einer langanhaltenden Erregbarkeitssteigerung des Motorkortex, die 24 Stunden nach Stimulation nachweisbar war. Die Länge der nachweisbaren Nacheffekte, die Abhängigkeit der Effekte anodaler tDCS von der NMDA-Rezeptoraktivität, sowie die repetitive Stimulationsform sprechen für eine ILTP-ähnliche Plastizitätsformierung.

Außerdem resultierte repetitive anodale tDCS mit einem stimulationsfreien Intervall von mehreren Stunden in der Ausbildung von homöostatischer Plastizität - denn nach der letzten Stimulation ließen sich keine eindeutig erregbarkeitssteigernden oder -vermindernden Effekte auf die kortikale Erregbarkeit nachweisen und insgesamt war das Aktivitätslevel gesunken.

Meine Überlegungen zu den ursächlichen Mechanismen für die neuroplastischen Veränderungen bei repetitiver anodaler Gleichstromstimulation mit unterschiedlich langen Pausen habe ich in den vorherigen Abschnitten bereits dargelegt. Einen definitiven Beweis für meine Vermutungen habe ich jedoch in dieser Arbeit nicht liefern können. Weitere Experimente werden die genaue Wirkungsweise (involvierte Rezeptoren, Transmitter, intrazelluläre Signalkaskaden) von repetitiver anodaler tDCS am Menschen aufklären müssen. Dabei könnte humanexperimentell der Einsatz von zentralwirksamen Medikamenten oder bildgebenden Verfahren zu neuen Erkenntnissen führen. Zusätzlich würde sich die Untersuchung repetitiver tDCS im Rattenmodell lohnen, um weitere zelluläre und molekulare Hintergründe aufzudecken.

Ein limitierender Faktor bei der Interpretation der Ergebnisse dieser Studie ist das Probandenkollektiv. Dieses hat einen nur geringen Stichprobenumfang von 15 Probanden und besitzt relativ homogene Eigenschaften (geringe Altersspanne; gesunde, junge Probanden; Rekrutierung ausschließlich aus dem studentischen Umfeld der Georg-August-Universität Göttingen), sodass die Übertragung der 
Ergebnisse auf ein inhomogenes, meist sehr viel älteres Patientenkollektiv mit bestehenden Veränderungen im Gehirnparenchym schwierig ist.

Zusätzlich wurden die Nachmessungen der MEP-Amplituden bei den repetitiven Stimulationsbedingungen mit relativ kurzen Pausen zu einem Zeitpunkt beendet, an dem diese noch deutlich über dem Niveau der Baseline waren, und es bleibt unklar, wann sie dieses Level wieder erreicht hätten. Daher wäre für spätere Studien die Beobachtung der Exzitabilitätsveränderung über einen noch längeren Zeitraum als 24 Stunden ein spannender Aspekt, um die Dauer der Nacheffekte exakt zu bestimmen.

Um die Ergebnisse meiner Dissertation in einem größeren Gesamtkontext zu betrachten, lohnt es sich, eine Verbindung zum Bereich der Lern- und Gedächtnisforschung, sowie zur medizinischen Klinik zu ziehen:

In den Neurowissenschaften gilt die synaptische Plastizitätsveränderung der Langzeitpotenzierung als Modell für Lern- und Gedächtnisprozesse (Costa-Mattioli et al. 2009; Huang YY et al. 1996; Lynch 2004; Neves et al. 2008). Erinnerungen des Kurzzeitgedächtnisses, die nur für wenige Minuten oder Stunden anhalten, werden durch eine erhöhte Ausschüttung von Neurotransmittern erzeugt, wohingegen Langzeiterinnerungen, die für viele Stunden, Tage, Monate oder Jahre existieren können, von neuer Proteinsynthese abhängig sind (Kandel 2001). Interessanterweise decken sich die Ergebnisse von Studien zu Gedächtnisleistungen mit denen dieses Experiments. So wurde beispielsweise die Erinnerungsleistung bei wiederholten, voneinander separierten Einheiten an Stelle von einem einmaligen Training verbessert (Callan und Schweighofer 2010; Philips und Carew 2009).

Dieses Experiment zeigt zum ersten Mal humanexperimentell, dass repetitive anodale tDCS in der Lage ist, I-LTP-ähnliche Plastizität im humanen Gehirn zu induzieren. In Anlehnung an diverse Verhaltensstudien zum Thema „Lernen und Gedächtnis“ deuten die Ergebnisse dieser Studie darauf hin, dass effektiveres Lernen beim Menschen durch Teilung der Lerninhalte in kleinere Abschnitte und deren Wiederholung in einem Abstand von wenigen Minuten erzielt werden könnte.

Im Hinblick auf den Einsatz der tDCS im klinischen Allag bei Erkrankungen des neuropsychiatrischen Formenkreises legen die Ergebnisse dieser Studie nahe, dass zur Ausbildung von noch länger anhaltenden und effektiveren 
Erregbarkeitsveränderungen des humanen Kortex der Einsatz von repetitiven Stimulationen gewinnbringend sein könnte. Die bislang durchgeführten Studien an Patienten mit Depression fanden in repetitiver Form an mehreren aufeinanderfolgenden Tagen statt und belegen bereits positive Effekte der Symptomlinderung durch tDCS (Fregni et al. 2006b). Die in dieser Arbeit vorgestellten Resultate deuten darauf hin, dass der Einsatz von mehreren transkraniellen Gleichstromstimulationen mit einem freien Intervall von wenigen Minuten ein neuer Ansatzpunkt in der klinischen Forschung sein könnte.

Interessant könnte für spätere Studien repetitiver transkranieller Gleichstromstimulation eine Modifikation des stimulationsfreien Intervalls sein, da in dieser Studie nur in begrenztem Umfang von wenigen Minuten oder mehreren Stunden eine Variation des zeitlichen Abstandes zwischen zwei Stimulationen vorgenommen wurde. Für nachfolgende Experimente ergibt sich in diesem Bereich noch ein großer Spielraum.

Außerdem sollten sich spätere Studien mit einer größeren Anzahl an repetitiven Stimulationen befassen um herauszufinden, ob dadurch noch effektivere Exzitabilitätssteigerungen $z u$ erreichen sind - wie es bereits im Tierexperiment nachgewiesen wurde (Trepel und Racine 1998) -, ob die Dauer der kortikalen Erregbarkeitssteigerung verlängert werden kann, oder aber, ob es zu entgegengesetzten Effekten bzw. zur Ausbildung von Homöostaseeffekten kommt. 


\section{Zusammenfassung}

Ziel der Arbeit war es, die Effektivität von repetitiver anodaler Gleichstromstimulation auf die Exzitabilität des humanen Motorkortex zu untersuchen. tDCS ist ein nichtinvasives, neuromodulatorisches Stimulationsverfahren des Gehirns. Kontinuierliche anodale Gleichstromstimulationsformen haben bis zu einer Stunde anhaltende kortikale Erregbarkeitssteigerungen des Motorkortex induzieren können. Die Auswirkungen repetitiver tDCS wurden bislang kaum erforscht.

$\mathrm{Da}$ tierexperimentell bereits gezeigt werden konnte, dass repetitive Stimulationsformen mit stimulationsfreien Intervallen von wenigen Minuten, langanhaltende ( $>3$ Stunden) synaptische Plastizitätsveränderungen auslösen, wollte ich in dieser Arbeit untersuchen, ob I-LTP-ähnliche Plastizität auch im Menschen durch repetitive anodale tDCS erzeugt werden kann.

In einem randomisierten Design erhielten 15 junge, gesunde Probanden wiederholt anodale tDCS von $1 \mathrm{~mA}$ über 13 Minuten, die durch unterschiedlich lange Pausen (3 Minuten, 20 Minuten, 3 Stunden oder 24 Stunden) voneinander getrennt waren und mit zwei Stimulationsformen 13-minütiger und 26-minütiger kontinuierlicher anodaler tDCS von $1 \mathrm{~mA}$ verglichen wurden.

In einem 2. Experiment wurden die zugrunde liegenden zellulären Mechanismen der Nacheffekte 26-minütiger kontinuierlicher anodaler tDCS von $1 \mathrm{~mA}$ an einer Subgruppe von 5 Probanden durch die orale Einnahme von $10 \mathrm{mg}$ des Kalziumkanalblockers Flunarizin analysiert. Die Veränderung der neuronalen Erregbarkeit wurde in beiden Experimenten mittels durch TMS generierter MEPAmplituden überwacht.

Die statistische Auswertung erfolgte über eine zweifaktorielle MesswiederholungsANOVA und den Fisher-LSD-Test.

Diese Arbeit konnte erstmals zeigen, dass repetitive anodale tDCS mit stimulationsfreien Intervallen von wenigen Minuten zu einer bis zu 24 Stunden anhaltenden Erregbarkeitssteigerung der Neurone des motorischen Kortex im Menschen führt. Aufgrund der Parallelen zu tierexperimentellen Resultaten kann erstmalig von einer I-LTP-ähnlichen Plastizitätsinduktion im Menschen gesprochen werden. Die genauen Mechanismen der in diesem Experiment nachgewiesenen kortikalen Erregbarkeitssteigerung konnten leider nicht aufgedeckt werden. Zusätzlich führte eine Verlängerung der kontinuierlichen anodalen tDCS von 13 Minuten auf 26 Minuten Dauer zu einer Erregbarkeitsverminderung der stimulierten 
Neurone, welche wahrscheinlich auf einen intrazellulären Kalziumüberschuss zurückzuführen ist und durch Flunarizin aufgehoben wurde. Außerdem resultierte repetitive anodale tDCS mit einer Pause von mehreren Stunden in der Ausbildung von homöostatischer Plastizität.

Zukünftige Studien werden sich mit der Untersuchung der genauen zellulären und molekularen Mechanismen der Plastizitätsinduktion durch repetitive anodale tDCS befassen müssen.

Schlussendlich weisen die Ergebnisse meiner Dissertation darauf hin, dass repetitive anodale tDCS mit einem stimulationsfreien Intervall von wenigen Minuten durch die nachgewiesenen effektiven und langanhaltenden Erregbarkeitsveränderungen in klinischen Studien an neuropsychiatrischen Patienten zur Therapieoptimierung führen könnte und auch Lernen durch Wiederholungen mit relativ kurzen Pausen möglicherweise effektiver gestaltet werden könnte. 


\section{Anhangsverzeichnis}

\subsection{Probandenaufklärung 1}

Informationen für die Teilnehmer der Studie: Neuroplastische Effekte der repetitiven anodalen transkraniellen Gleichstromstimulation über dem motorischen Kortex.

Sehr geehrte Teilnehmerin, sehr geehrter Teilnehmer!

Wir bedanken uns für Ihr Interesse an der o.g. Studie und möchten Ihnen den Ablauf der Studie anhand dieses Informationsbogens erläutern.

Diese Studie umfasst 6 Sitzungen, in denen mittels durch die Kopfhaut und den Schädel (transkraniell) gegebener Magnetimpulse mit Einzel- und Doppelstimulation Erregungs- und Hemmungsvorgänge im Bereich Ihrer motorischen Hirnrinde gemessen werden sollen.

Die transkranielle Magnetstimulation ist eine nicht-invasive und schmerzfreie Untersuchungsmethode. Die bereits vorliegenden, umfangreichen Erfahrungen haben gezeigt, dass dieses Verfahren risiko- und nebenwirkungsarm ist, wenn die Ausschlusskriterien beachtet werden (siehe unten). In seltenen Fällen ist mit dem Auftreten von Müdigkeit und Kopfschmerzen zu rechnen. Die Stimulation erfolgt mittels einer Reizspule, die auf Ihren Kopf aufgelegt wird. Bei überschwelliger Stimulation werden Sie eine leichte unwillkürliche Zuckung in einigen Muskeln der Hand verspüren. Mit Oberflächenelektroden werden wir diese Zuckungen von einem Ihrer Handmuskeln registrieren.

Zusätzlich werden in allen Sitzungen zwei Elektroden auf Ihrer Kopfhaut angebracht. Über diese Elektroden fließt während der Untersuchung jeweils für 13 bzw. 26 Minuten ein schwacher Gleichstrom, dessen Auswirkung auf die Erregbarkeit Ihres Gehirns untersucht werden soll. In 4 der 6 Sitzungen wird nach 13-minütigem Stromfluss für 3 Minuten, 20 Minuten, 3 Stunden oder 24 Stunden pausiert und anschließend eine wiederholte 13-minütige Stimulation stattfinden. Der schwache Stromfluss ist für Sie nicht oder allenfalls sehr geringfügig wahrnehmbar. Dieses ebenfalls nicht-invasive und schmerzfreie Verfahren wurde bereits in mehreren Untersuchungen angewendet und hat sich als risiko- und nebenwirkungsarm 
erwiesen. Selten und lediglich nach kontinuierlicher Gleichstromapplikation über Stunden traten leichte Kopfschmerzen und Hautreizungen im Bereich der Elektroden auf, die relativ schnell vollständig reversibel waren.

Die Studie umfasst voraussichtlich 6 Termine von je 3-3,5 Stunden Dauer. Zwischen den einzelnen Sitzungen muss jeweils mindestens eine Woche pausiert werden. Wir streben an, dass jeder Proband/ jede Probandin alle Untersuchungen durchläuft.

Selbstverständlich ist es Ihnen jederzeit möglich, ohne Angabe von Gründen und ohne dass Ihnen hieraus Nachteile entstehen, von der Teilnahme an der Studie zurückzutreten.

Falls einer der folgenden Punkte auf Sie zutrifft, ist eine Teilnahme an der Studie nicht möglich:

1. Herzschrittmacher

2. Metallimplantate im Kopfbereich (in den Kopfbereich eingesetztes Metall, z.B. Clips nach Operation eines intrazerebralen Aneurysmas (Gefäßaussackung im Bereich der Hirngefäße)), Implantation einer künstlichen Hörschnecke

3. Alter $<18$ oder $>75$ Jahre

4. Hinweise auf eine chronische oder Residuen (Reste) einer neurologischen Erkrankung (Erkrankung des Nervensystems) in der Vorgeschichte

5. Intrazerebrale Ischämien (Mangeldurchblutung des Gehirns, Schlaganfall, Blutungen) in der Vorgeschichte

6. Hinweise auf epileptische Anfälle in der Vorgeschichte

7. Vorliegen einer gravierenden internistischen (Erkrankungen der inneren Organe) oder psychiatrischen (seelische Erkrankung) Vorerkrankung, insbesondere Schizophrenie (Spaltungsirresein) oder Manie (Erkrankung des Gemüts mit gehobener Stimmung)

8. Schwangerschaft oder Stillperiode

9. Drogen- und/oder Alkoholabhängigkeit

10. Rezeptive oder globale Aphasie (Störung des Sprachverständnisses bzw. zusätzlich des Sprechens)

11. Teilnahme an einer anderen klinischen Prüfung innerhalb der letzten 8 Wochen 
Die aus der Studie gewonnenen Daten werden anonymisiert wissenschaftlich ausgewertet. Alle Erfordernisse des Datenschutzes werden dabei beachtet.

\subsection{Probandenaufklärung 2}

Informationen für die Teilnehmer über die Untersuchung: Pharmakologische Beeinflussbarkeit der Nacheffekte schwacher transkranieller Gleichstromstimulation auf die kortikale Exzitabilität

Sehr geehrte Teilnehmerin, sehr geehrter Teilnehmer!

Wir möchten Sie bitten, an einer wissenschaftlichen Untersuchung teilzunehmen. Durch diese Studie entsteht Innen kein direkter Nutzen, die Ergebnisse können aber möglicherweise in der Zukunft geeignet sein, neue therapeutische Verfahren zur Behandlung von Erkrankungen zu entwickeln.

Diese Studie umfasst 1 Sitzung, in der mittels durch die Kopfhaut und den Schädel (transkraniell) gegebener Magnetimpulse mit Einzel- und Doppelstimulation Erregungs- und Hemmungsvorgänge im Bereich Ihrer motorischen Hirninde gemessen werden sollen.

Die transkranielle Magnetstimulation ist eine nicht-invasive und schmerzfreie Untersuchungsmethode. Die bereits vorliegenden, umfangreichen Erfahrungen haben gezeigt, dass dieses Verfahren risiko- und nebenwirkungsarm ist, wenn die Ausschlusskriterien beachtet werden (siehe unten). In seltenen Fällen ist mit Auftreten von Müdigkeit und Kopfschmerzen zu rechnen. Die Stimulation erfolgt mittels einer Reizspule, die auf Ihren Kopf aufgelegt wird. Bei überschwelliger Stimulation werden Sie eine leichte unwillkürliche Zuckung in einigen Muskeln der Hand verspüren. Mit Oberflächenelektroden werden wir von einem Ihrer Handmuskeln diese Zuckungen registrieren.

Zusätzlich werden in der Sitzung zwei Elektroden auf Ihrer Kopfhaut angebracht. Über diese Elektroden fließt während der Untersuchung für 26 Minuten ein schwacher Gleichstrom, dessen Auswirkung auf die Erregbarkeit Ihres Gehirns untersucht werden soll. Der schwache Stromfluss ist für Sie nicht oder allenfalls sehr geringfügig wahrnehmbar. Dieses ebenfalls nicht-invasive und schmerzfreie Verfahren wurde bereits in mehreren Untersuchungen angewendet und hat sich als 
risiko- und nebenwirkungsarm erwiesen. Selten und lediglich nach kontinuierlicher Gleichstromapplikation über Stunden traten leichte Kopfschmerzen und Hautreizungen im Bereich der Elektroden auf, die relativ schnell vollständig reversibel waren.

Darüber hinaus ist die Anwendung eines Medikaments geplant, um dessen Einfluss auf die gemessenen Parameter zu bestimmen. Angewandt wird das Medikament Flunarizin als Tablette. Dieses Medikament ist in seiner Wirkung und Nebenwirkung gut bekannt. Die Einnahme soll kurz vor Beginn der Untersuchung erfolgen. Wir weisen darauf hin, dass das Führen eines Kraftfahrzeuges oder das Arbeiten an selbst- bzw. fremdgefährdenden Maschinen nach Einnahme des Medikaments bis zum Morgen des Tages nach der Untersuchung unterbleiben muss, da eine deutliche Beeinträchtigung der Fahrtüchtigkeit vorliegen kann. An sonstigen Nebenwirkungen können auftreten:

Flunarizin: Benommenheit, Müdigkeit, Gewichtszunahme, depressive Verstimmung, Bewegungsstörungen, Sodbrennen, Übelkeit, Magenschmerzen, Schlafzustände, Angst, Kopfschmerzen, Mundtrockenheit, Muskelschmerzen, Hautrötung.

Die Studie umfasst voraussichtlich 1 Termin von 3-3,5 Stunden Dauer.

Selbstverständlich ist es Ihnen jederzeit möglich, ohne Angabe von Gründen und ohne, dass Ihnen hieraus Nachteile entstehen, von der Teilnahme an der Studie zurückzutreten.

Falls einer der folgenden Punkte auf Sie zutrifft, ist eine Teilnahme an der Studie nicht möglich:

1. Herzschrittmacher

2. Metallimplantate im Kopfbereich (in den Kopfbereich eingesetztes Metall, z.B. Clips nach Operation eines intrazerebralen Aneurysmas (Gefäßaussackung im Bereich der Hirngefäße)), Implantation einer künstlichen Hörschnecke

3. Alter $<18$ oder $>75$ Jahre 
4. Hinweise auf eine chronische oder Residuen (Reste) einer neurologischen Erkrankung (Erkrankung des Nervensystems) in der Vorgeschichte

5. Intrazerebrale Ischämien (Mangeldurchblutung des Gehirns, Schlaganfall, Blutungen) in der Vorgeschichte

6. Hinweise auf epileptische Anfälle in der Vorgeschichte

7. Vorliegen einer gravierenden internistischen (Erkrankung der inneren Organe) oder psychiatrischen (seelische Erkrankung) Vorerkrankung, insbesondere Schizophrenie (Spaltungsirresein) oder Manie (Erkrankung des Gemüts mit gehobener Stimmung)

8. Schwangerschaft oder Stillperiode

9. Drogen- und/oder Alkoholabhängigkeit

10. Rezeptive oder globale Aphasie (Störung des Sprachverständnisses bzw. zusätzlich des Sprechens)

11. Teilnahme an einer anderen klinischen Prüfung innerhalb der letzten 8 Wochen

Die aus der Studie gewonnenen Daten werden anonymisiert wissenschaftlich ausgewertet. Alle Erfordernisse des Datenschutzes werden dabei beachtet. 


\subsection{Einverständniserklärung}

Einverständniserklärung zur Teilnahme an der Untersuchung:

Einfluss schwacher transkranieller Gleichstromstimulation auf die zerebrale Exzitabilität und dessen Modulierbarkeit durch transkranielle Magnetstimulation

Herr/Frau (Dr.) hat mir heute anhand der Hinweise auf dem Informationsbogen für Teilnehmer an o.g. Studie die Durchführung der Untersuchung erläutert.

Ich habe diesbezüglich keine weiteren Fragen mehr und willige hiermit in die dargestellten Untersuchungen ein. Alle mich interessierenden Fragen wurden ausreichend beantwortet. Ich erkläre darüber hinaus, dass ich alle Angaben zur Krankengeschichte wahrheitsgemäß gemacht habe.

Mir ist bekannt, dass ich jederzeit ohne Angaben von Gründen die weitere Untersuchung ablehnen bzw. mein Einverständnis widerrufen kann, ohne dass mir daraus Nachteile entstehen.

Ich bin bereit, an der Studie teilzunehmen.

Alle Befunde sind vertraulich und werden unter strikter Einhaltung des Datenschutzes gehandhabt.

Eine Kopie dieser Erklärung wurde mir überreicht.

Göttingen, den

Name des aufklärenden Arztes (in Druckbuchstaben) und Unterschrift

Name des Patienten (in Druckbuchstaben) und Unterschrift 


\subsection{Weitere Abbildungen und Tabellen}

Abbildung 5: Versuchsablauf der Experimente 1 und 2




Diese Graphik beschreibt die Versuchsdurchführung der 2 Experimente dieser Arbeit in anschaulicher Form. Im Experiment 1 wurden 4 verschiedene Stimulationsbedingungen 13-minütiger repetitiver anodaler Gleichstromstimulation mit relativ kurzen Pausen (3 Minuten, 20 Minuten) oder relativ langen Pausen (3 Stunden, 24 Stunden) durchgeführt und mit den Stimulationen kontinuierlicher anodaler tDCS von 13 Minuten und 26 Minuten verglichen. Die kortikalen Erregbarkeitsveränderungen wurden mittels Einzelpuls-TMS-Messungen zu mehreren Zeitpunkten am Stimulationstag und am Folgetag für die Zeitpunkte Morgen ( $n m=$ next morning), Mittag (na= next afternoon) und Abend (ne= next evening) überwacht. Eine Ausnahme davon stellte die Sitzung kontinuierlicher 13minütiger anodaler tDCS dar, bei der die Nachmessungen nur bis zum Abend des Stimulationstages durchgeführt wurden. Im 2. Experiment wurden die Effekte des Kalziumkanalblockers Flunarizin (FLU= $10 \mathrm{mg}$ Flunarizin) auf eine 26-minütige kontinuierliche anodale tDCS von $1 \mathrm{~mA}$ untersucht. Nach Aufzeichnung der 1. Ausgangsmessung vor tDCS mittels TMS erfolgte die orale Einnahme des Medikaments. Nach 2 Stunden wurde die Ausgangsmessung (Baseline 2) mit gleicher TMS-Intensität wiederholt und im Bedarfsfall angepasst (Baseline 3). Nach Abschluss der anodalen Gleichstromstimulation erfolgte die Aufzeichnung der MEPMessungen bis zur 120. Minute nach Stimulationsende. 
Tabelle 4: Erhobene Messwerte der Experimente 1 und 2 vor und nach tDCS

Die nachfolgenden Tabellen beinhalten für jede Sitzungsbedingung der Experimente 1 und 2 die jeweils erhobenen Mittelwerte der MEP-Amplituden für die Ausgangsmessung vor tDCS und die Mittelwerte der MEP-Amplituden für jeden Messzeitpunkt nach erfolgter tDCS mit dazugehöriger Standardabweichung und dem Standardfehler. Die Stimulationsbedingungen sind in abgekürzter Form mit dazwischenliegender variabler Pausen von $\min =$ Minuten oder $h=$ Stunden, sowie mit oder ohne Medikation ( $F L U=10 \mathrm{mg}$ Flunarizin) dargestellt. Baseline= Ausgangsmessung vor tDCS; $n=$ Stichprobenumfang; $S D=$ Standardabweichung; $S E M=$ Standardfehler; se= Zeitpunkt Abend des Stimulationstages; $n m=$ Zeitpunkt Morgen des Folgetages der letzten anodaler tDCS; na= Zeitpunkt Nachmittag des Folgetages der letzten anodalen tDCS; ne= Zeitpunkt Abend des Folgetages der letzten anodalen $t D C S$.

\section{Experiment 1}

\begin{tabular}{|l|l|l|l|l|}
\hline $\mathbf{1 3 - 0 - 0}$ & $\mathrm{n}$ & Mittelwert & SD & SEM \\
\hline Baseline & 15 & 0,947116 & 0,102004 & 0,026337 \\
\hline 0 min & 15 & 1,097333 & 0,202715 & 0,052341 \\
\hline 5 min & 15 & 1,184072 & 0,339569 & 0,087676 \\
\hline 10 min & 15 & 1,191246 & 0,260987 & 0,067387 \\
\hline 15 in & 15 & 1,16438 & 0,19382 & 0,050044 \\
\hline 20 min & 15 & 1,196186 & 0,325604 & 0,084071 \\
\hline 25 in & 15 & 1,228368 & 0,356511 & 0,092051 \\
\hline 30 min & 15 & 1,130042 & 0,269125 & 0,069488 \\
\hline 60 min & 15 & 1,168876 & 0,196414 & 0,050714 \\
\hline 90 min & 15 & 1,034747 & 0,275077 & 0,071025 \\
\hline 120 min & 15 & 1,008987 & 0,171233 & 0,044212 \\
\hline se & 15 & 1,058561 & 0,199573 & 0,051529 \\
\hline
\end{tabular}

\begin{tabular}{|l|l|l|l|l|}
\hline $\mathbf{1 3 - 0}-\mathbf{1 3}$ & $\mathrm{n}$ & Mittelwert & SD & SEM \\
\hline Baseline & 15 & 1,002919 & 0,104916 & 0,027089 \\
\hline $0 \mathrm{~min}$ & 15 & 0,831925 & 0,228596 & 0,059023 \\
\hline $5 \mathrm{~min}$ & 15 & 0,820944 & 0,30525 & 0,078815 \\
\hline $10 \mathrm{~min}$ & 15 & 0,867331 & 0,355914 & 0,091897 \\
\hline $15 \mathrm{~min}$ & 15 & 0,767968 & 0,260994 & 0,067388 \\
\hline $20 \mathrm{~min}$ & 15 & 0,77455 & 0,26815 & 0,069236 \\
\hline $25 \mathrm{~min}$ & 15 & 0,791831 & 0,263617 & 0,068066 \\
\hline $30 \mathrm{~min}$ & 15 & 0,842813 & 0,339368 & 0,087624 \\
\hline $60 \mathrm{~min}$ & 15 & 0,846862 & 0,273156 & 0,070529 \\
\hline $90 \mathrm{~min}$ & 15 & 0,894056 & 0,367495 & 0,094887 \\
\hline $120 \mathrm{~min}$ & 15 & 0,828754 & 0,200385 & 0,051739 \\
\hline $\mathrm{se}$ & 12 & 0,983896 & 0,466558 & 0,120465 \\
\hline $\mathrm{nm}$ & 11 & 0,937271 & 0,316893 & 0,095547 \\
\hline $\mathrm{na}$ & 11 & 0,972792 & 0,262851 & 0,079252 \\
\hline
\end{tabular}




\begin{tabular}{|l|l|l|l|l|}
\hline ne & 9 & 0,913452 & 0,19317 & 0,06439 \\
\hline
\end{tabular}

\begin{tabular}{|l|l|l|l|l|}
\hline 13-3 min-13 & $\mathrm{n}$ & Mittelwert & $\mathrm{SD}$ & SEM \\
\hline Baseline & 15 & 0,947157 & 0,129377 & 0,033405 \\
\hline 0 min & 15 & 1,222367 & 0,389281 & 0,100512 \\
\hline $5 \mathrm{~min}$ & 15 & 1,145491 & 0,364861 & 0,094207 \\
\hline $10 \mathrm{~min}$ & 15 & 1,120422 & 0,360979 & 0,093204 \\
\hline $15 \mathrm{~min}$ & 15 & 1,149653 & 0,332844 & 0,08594 \\
\hline $20 \mathrm{~min}$ & 15 & 1,195186 & 0,397437 & 0,102618 \\
\hline $25 \mathrm{~min}$ & 15 & 1,194691 & 0,462689 & 0,119466 \\
\hline $30 \mathrm{~min}$ & 15 & 1,095721 & 0,384344 & 0,099237 \\
\hline $60 \mathrm{~min}$ & 15 & 1,059127 & 0,406814 & 0,105039 \\
\hline $90 \mathrm{~min}$ & 15 & 1,003863 & 0,268787 & 0,069401 \\
\hline $120 \mathrm{~min}$ & 15 & 1,02164 & 0,346549 & 0,089479 \\
\hline $\mathrm{se}$ & 15 & 1,348554 & 0,56394 & 0,098 \\
\hline $\mathrm{nm}$ & 14 & 1,298183 & 0,360971 & 0,096473 \\
\hline na & 15 & 1,209187 & 0,328921 & 0,084927 \\
\hline ne & 14 & 1,216916 & 0,270167 & 0,072205 \\
\hline
\end{tabular}

\begin{tabular}{|l|l|l|l|l|}
\hline $\mathbf{1 3 - 2 0}$ min-13 & $\mathrm{n}$ & Mittelwert & $\mathrm{SD}$ & SEM \\
\hline Baseline & 15 & 0,890144 & 0,099445 & 0,025677 \\
\hline $0 \mathrm{~min}$ & 15 & 0,0981401 & 0,325705 & 0,084097 \\
\hline $5 \mathrm{~min}$ & 15 & 1,148897 & 0,332888 & 0,085951 \\
\hline $10 \mathrm{~min}$ & 15 & 1,04897 & 0,283288 & 0,073145 \\
\hline $15 \mathrm{~min}$ & 15 & 1,112816 & 0,330177 & 0,085251 \\
\hline $20 \mathrm{~min}$ & 15 & 1,164604 & 0,486247 & 0,125548 \\
\hline $25 \mathrm{~min}$ & 15 & 1,185234 & 0,345407 & 0,089184 \\
\hline $30 \mathrm{~min}$ & 15 & 1,224807 & 0,504067 & 0,130149 \\
\hline $60 \mathrm{~min}$ & 15 & 1,247909 & 0,525372 & 0,13565 \\
\hline $90 \mathrm{~min}$ & 15 & 1,194826 & 0,440608 & 0,113764 \\
\hline $120 \mathrm{~min}$ & 15 & 1,023558 & 0,459661 & 0,118684 \\
\hline $\mathrm{se}$ & 15 & 1,305848 & 0,564729 & 0,145813 \\
\hline $\mathrm{mm}$ & 15 & 1,28788 & 0,822004 & 0,12 \\
\hline $\mathrm{na}$ & 15 & 1,353261 & 0,627022 & 0,1 \\
\hline $\mathrm{ne}$ & 15 & 1,456582 & 0,688229 & 0,114 \\
\hline
\end{tabular}

\begin{tabular}{|l|l|l|l|l|}
\hline $\mathbf{1 3 - 3 h - 1 3}$ & $\mathrm{n}$ & Mittelwert & $\mathrm{SD}$ & SEM \\
\hline Baseline & 15 & 0,950861 & 0,134524 & 0,034734 \\
\hline $0 \mathrm{~min}$ & 15 & 0,969896 & 0,254801 & 0,065789 \\
\hline $5 \mathrm{~min}$ & 15 & 0,918775 & 0,231103 & 0,05967 \\
\hline $10 \mathrm{~min}$ & 15 & 1,034636 & 0,310325 & 0,080126 \\
\hline $15 \mathrm{~min}$ & 15 & 0,961719 & 0,327898 & 0,084663 \\
\hline $20 \mathrm{~min}$ & 15 & 0,888445 & 0,161001 & 0,04157 \\
\hline $25 \mathrm{~min}$ & 15 & 0,999142 & 0,353257 & 0,091211 \\
\hline
\end{tabular}




\begin{tabular}{|l|l|l|l|l|}
\hline $30 \mathrm{~min}$ & 15 & 0,885788 & 0,312572 & 0,080706 \\
\hline $60 \mathrm{~min}$ & 15 & 0,92126 & 0,422739 & 0,109151 \\
\hline $90 \mathrm{~min}$ & 15 & 1,040116 & 0,519984 & 0,134259 \\
\hline $120 \mathrm{~min}$ & 15 & 1,142928 & 0,409725 & 0,10579 \\
\hline se & 15 & 1,031109 & 0,259711 & 0,067057 \\
\hline nm & 15 & 0,962149 & 0,35738 & 0,092275 \\
\hline na & 15 & 1,162057 & 0,552093 & 0,1 \\
\hline ne & 15 & 0,986175 & 0,357045 & 0,092188 \\
\hline
\end{tabular}

\begin{tabular}{|l|l|l|l|l|}
\hline 13-24h-13 & $\mathrm{n}$ & Mittelwert & $\mathrm{SD}$ & $\mathrm{S} E M$ \\
\hline Baseline & 15 & 0,998933 & 0,116622 & 0,030112 \\
\hline $0 \mathrm{~min}$ & 15 & 0,934405 & 0,194247 & 0,050154 \\
\hline $5 \mathrm{~min}$ & 15 & 0,870735 & 0,195004 & 0,05035 \\
\hline $10 \mathrm{~min}$ & 15 & 0,942056 & 0,276753 & 0,071457 \\
\hline $15 \mathrm{~min}$ & 15 & 1,008246 & 0,348776 & 0,090054 \\
\hline $20 \mathrm{~min}$ & 15 & 0,980388 & 0,396865 & 0,10247 \\
\hline $25 \mathrm{~min}$ & 15 & 1,013118 & 0,370355 & 0,095625 \\
\hline $30 \mathrm{~min}$ & 15 & 0,991518 & 0,363218 & 0,093783 \\
\hline $60 \mathrm{~min}$ & 15 & 1,012248 & 0,340904 & 0,088021 \\
\hline $90 \mathrm{~min}$ & 15 & 0,973448 & 0,30849 & 0,079652 \\
\hline $120 \mathrm{~min}$ & 15 & 1,055712 & 0,385019 & 0,099411 \\
\hline se & 15 & 1,069069 & 0,335737 & 0,086687 \\
\hline nm & 14 & 1,113782 & 0,479977 & 0,098 \\
\hline na & 14 & 1,146168 & 0,590152 & 0,1 \\
\hline ne & 14 & 0,974251 & 0,308943 & 0,082568 \\
\hline
\end{tabular}

\section{Experiment 2}

\begin{tabular}{|l|l|l|l|l|}
\hline $\mathbf{1 3 - 0 - 1 3}$ Kontrolle & $\mathrm{n}$ & Mittelwert & $\mathrm{SD}$ & $\mathrm{SEM}$ \\
\hline Baseline & 5 & 1,092 & 0,058 & 0,033 \\
\hline $0 \mathrm{~min}$ & 5 & 0,94609154 & 0,1768464 & 0,07908811 \\
\hline $5 \mathrm{~min}$ & 5 & 0,77138362 & 0,18307637 & 0,08187424 \\
\hline $10 \mathrm{~min}$ & 5 & 0,78614709 & 0,13523443 & 0,06047868 \\
\hline $15 \mathrm{~min}$ & 5 & 0,75931882 & 0,18744588 & 0,08382879 \\
\hline $20 \mathrm{~min}$ & 5 & 0,67101973 & 0,14308854 & 0,06399114 \\
\hline $25 \mathrm{~min}$ & 5 & 0,73344322 & 0,15774431 & 0,0705454 \\
\hline $30 \mathrm{~min}$ & 5 & 0,733953917 & 0,172207857 & 0,077013695 \\
\hline $60 \mathrm{~min}$ & 5 & 0,797790059 & 0,089233523 & 0,039906445 \\
\hline $90 \mathrm{~min}$ & 5 & 0,792454251 & 0,154437074 & 0,06906636 \\
\hline $120 \mathrm{~min}$ & 5 & 0,80031985 & 0,2010098 & 0,08989431 \\
\hline
\end{tabular}




\begin{tabular}{|l|l|l|l|l|}
\hline $\mathbf{1 3 - 0 - 1 3 + \text { FLU }}$ & $\mathrm{n}$ & Mittelwert & $\mathrm{SD}$ & $\mathrm{SEM}$ \\
\hline Baseline & 5 & 1,008 & 0,105 & 0,061 \\
\hline $0 \mathrm{~min}$ & 5 & 1,126013382 & 0,322706174 & 0,144318588 \\
\hline $5 \mathrm{~min}$ & 5 & 1,063630387 & 0,259776009 & 0,116175363 \\
\hline $10 \mathrm{~min}$ & 5 & 1,04099687 & 0,315112939 & 0,14092279 \\
\hline $15 \mathrm{~min}$ & 5 & 0,904262879 & 0,09662419 & 0,043211651 \\
\hline $20 \mathrm{~min}$ & 5 & 0,980183575 & 0,225540614 & 0,100864829 \\
\hline $25 \mathrm{~min}$ & 5 & 1,017562363 & 0,275562633 & 0,123235356 \\
\hline $30 \mathrm{~min}$ & 5 & 1,074216343 & 0,250449533 & 0,112004436 \\
\hline $60 \mathrm{~min}$ & 5 & 1,033636557 & 0,242983979 & 0,108665739 \\
\hline $90 \mathrm{~min}$ & 5 & 0,868041009 & 0,209835506 & 0,093841291 \\
\hline $120 \mathrm{~min}$ & 5 & 0,852867235 & 0,219246414 & 0,098049977 \\
\hline
\end{tabular}

\section{Tabelle 5: p-Werte der Fisher-LSD-Test-Berechnung für das Experiment 1}

Dargestellt sind die Ergebnisse der Fisher-LSD-Test-Berechnung zur Überprüfung signifikanter Unterschiede zwischen den jeweiligen Sitzungen kontinuierlicher und repetitiver anodaler tDCS zum jeweils angegebenen Zeitpunkt nach erfolgter Stimulation. Die 6 Stimulationsbedingungen wurden untereinander verglichen. Die Sitzungen sind in abgekürzter Form dargestellt. Pausen zwischen repetitiver tDCS sind in $\min =$ Minuten oder $h=$ Stunden angegeben. Signifikante $p$-Werte von $\leq 0,05$ sind durch * markiert. se= Zeitpunkt Abend des Stimulationstages.

\begin{tabular}{|c|c|c|c|c|c|}
\hline & $13-0-13$ & 13-3min-13 & $13-20 \mathrm{~min}-13$ & 13-3h-13 & $13-24 h-13$ \\
\hline $13-0-0$ & p-Wert & p-Wert & p-Wert & p-Wert & p-Wert \\
\hline $0 \mathrm{~min}$ & $0,029^{*}$ & 0,304 & 0.340 & 0,295 & 0,180 \\
\hline $5 \mathrm{~min}$ & $0,003^{*}$ & 0,751 & 0,772 & $0,029^{*}$ & $0,010^{*}$ \\
\hline $10 \mathrm{~min}$ & $0,008^{*}$ & 0,560 & 0,242 & 0,198 & $0,041^{*}$ \\
\hline $15 \mathrm{~min}$ & $0,001^{*}$ & 0,904 & 0,672 & 0,096 & 0,199 \\
\hline $20 \mathrm{~min}$ & $0,001^{*}$ & 0,993 & 0,795 & $0,012^{*}$ & 0,076 \\
\hline $25 \mathrm{~min}$ & $0,000^{*}$ & 0,782 & 0,723 & 0,060 & 0,077 \\
\hline $30 \mathrm{~min}$ & $0,018^{*}$ & 0,778 & 0,436 & $0,045^{*}$ & 0,255 \\
\hline $60 \mathrm{~min}$ & $0,008^{*}$ & 0,367 & 0,516 & $0,042^{*}$ & 0,198 \\
\hline $90 \min$ & 0,247 & 0,800 & 0,188 & 0,965 & 0,614 \\
\hline $120 \mathrm{~min}$ & 0,138 & 0,917 & 0,905 & 0.271 & 0,701 \\
\hline se & 0.539 & $0,017^{\star}$ & $0,042^{*}$ & 0,821 & 0,931 \\
\hline
\end{tabular}




\begin{tabular}{|c|c|c|c|c|c|}
\hline & $13-0-13$ & 13-3min-13 & $\begin{array}{l}13-20 \mathrm{~min}- \\
13\end{array}$ & $13-3 h-13$ & $13-24 h-13$ \\
\hline $13-0-13$ & p-Wert & p-Wert & p-Wert & p-Wert & p-Wert \\
\hline $0 \min$ & - & $0,001^{*}$ & 0,219 & 0,256 & 0,400 \\
\hline $5 \min$ & - & $0,008^{*}$ & $0,007^{*}$ & 0,421 & 0,682 \\
\hline $10 \mathrm{~min}$ & - & $0,038^{*}$ & 0,135 & 0,169 & 0,539 \\
\hline $15 \mathrm{~min}$ & - & $0,002^{*}$ & $0,005^{\star}$ & 0,111 & $0,048^{*}$ \\
\hline $20 \mathrm{~min}$ & - & $0,001^{*}$ & $0,001^{*}$ & 0,349 & 0,091 \\
\hline $25 \mathrm{~min}$ & - & $0,001^{*}$ & $0,000^{*}$ & 0,088 & 0,069 \\
\hline $30 \mathrm{~min}$ & - & $0,038^{*}$ & $0,002^{*}$ & 0,724 & 0,222 \\
\hline $60 \mathrm{~min}$ & - & 0,081 & $0,001^{*}$ & 0,541 & 0,174 \\
\hline $90 \mathrm{~min}$ & - & 0,367 & $0,014^{*}$ & 0,230 & 0,514 \\
\hline $120 \mathrm{~min}$ & - & 0,113 & 0,109 & $0,024^{*}$ & 0,062 \\
\hline 13-3min-13 & - & - & - & - & - \\
\hline $0 \min$ & - & - & $0,048^{*}$ & $0,038^{*}$ & $0,018^{*}$ \\
\hline $5 \min$ & - & - & 0,978 & 0,062 & $0,024^{*}$ \\
\hline $10 \mathrm{~min}$ & - & - & 0,557 & 0,481 & 0,143 \\
\hline $15 \min$ & - & - & 0,762 & 0,122 & 0,245 \\
\hline $20 \mathrm{~min}$ & - & - & 0,801 & $0,012^{*}$ & 0,078 \\
\hline $25 \mathrm{~min}$ & 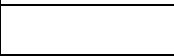 & - & 0,938 & 0,108 & 0,136 \\
\hline $30 \mathrm{~min}$ & - & - & 0,289 & 0,084 & 0.392 \\
\hline $60 \mathrm{~min}$ & - & - & 0,121 & 0,257 & 0,700 \\
\hline $90 \min$ & - & - & 0,117 & 0,766 & 0,802 \\
\hline $120 \mathrm{~min}$ & - & - & 0,987 & 0,319 & 0,779 \\
\hline $\begin{array}{l}13-20 \mathrm{~min}- \\
13\end{array}$ & - & - & - & - & - \\
\hline $0 \mathrm{~min}$ & - & - & - & 0,926 & 0,699 \\
\hline $5 \min$ & - & - & - & 0.031 & 0,196 \\
\hline $10 \mathrm{~min}$ & - & - & - & 0,906 & 0,379 \\
\hline $15 \mathrm{~min}$ & - & - & - & 0,214 & 0,390 \\
\hline $20 \mathrm{~min}$ & - & - & - & 0,023 & 0,130 \\
\hline $25 \mathrm{~min}$ & - & - & - & 0,126 & 0,908 \\
\hline $30 \mathrm{~min}$ & - & - & - & $0,005^{*}$ & 0,055 \\
\hline $60 \mathrm{~min}$ & - & - & - & $0,007^{\star}$ & 0,053 \\
\hline $90 \min$ & - & - & - & 0,203 & 0,069 \\
\hline $120 \min$ & - & - & - & 0,326 & 0,791 \\
\hline $13-3 h-13$ & - & - & - & - & - \\
\hline $0 \min$ & - & - & - & - & 0,769 \\
\hline $5 \mathrm{~min}$ & - & - & - & - & 0,693 \\
\hline $10 \mathrm{~min}$ & - & - & - & - & 0,446 \\
\hline $15 \mathrm{~min}$ & - & - & - & - & 0,702 \\
\hline $20 \mathrm{~min}$ & - & - & - & - & 0,450 \\
\hline $25 \mathrm{~min}$ & - & - & - & - & 0,908 \\
\hline $30 \mathrm{~min}$ & - & - & - & - & 0,385 \\
\hline $60 \mathrm{~min}$ & - & - & - & - & 0,541 \\
\hline
\end{tabular}




\begin{tabular}{|l|l|l|l|l|l|}
\hline $90 \min$ & - & - & - & - & 0,584 \\
\hline $120 \min$ & - & - & - & - & 0,099 \\
\hline
\end{tabular}

Tabelle 6: p-Werte der Fisher-LSD-Test-Berechnung für das Experiment 2

Dargestellt sind die Ergebnisse der Fisher-LSD-Test-Berechnung zur Überprüfung signifikanter Unterschiede zwischen den jeweiligen Sitzungen kontinuierlicher 26minütiger anodaler tDCS mit und ohne medikamentösen Einfluss durch Flunarizin zum jeweils angegebenen Zeitpunkt nach erfolgter Stimulation. Die Sitzungen sind in abgekürzter Form dargestellt. FLU $10 \mathrm{mg}$ Flunarizin. Signifikante $p$-Werte von $\leq$ 0,05 sind durch * markiert.

\begin{tabular}{|l|l|}
\hline & $\mathbf{1 3 - 0 - 1 3 ~ F L U ~}$ \\
\hline $\mathbf{1 3 - 0 - 1 3}$ & $\mathbf{p}-$ Wert \\
\hline $0 \mathrm{~min}$ & 0,382 \\
\hline $5 \mathrm{~min}$ & $0,047^{\star}$ \\
\hline $10 \mathrm{~min}$ & 0,077 \\
\hline $15 \mathrm{~min}$ & $0,042^{*}$ \\
\hline $20 \mathrm{~min}$ & $0,013^{*}$ \\
\hline $25 \mathrm{~min}$ & 0,055 \\
\hline $30 \mathrm{~min}$ & $0,025^{*}$ \\
\hline $60 \mathrm{~min}$ & 0,073 \\
\hline $90 \mathrm{~min}$ & 0,554 \\
\hline $120 \mathrm{~min}$ & 0,722 \\
\hline & \\
\hline
\end{tabular}




\section{Literaturverzeichnis}

Abraham WC, Mason-Parker SE, Bear MF, Webb S \& Tate WP (2001): Heterosynaptic metaplasticity in the hippocampus in vivo: a BCM-like modifiable threshold for LTP. Proc Natl Acad Sci USA; 98: 10924-10929

Antal A, Nitsche MA, Paulus W (2001): External modulation of visual perception in humans. Neuroreport; 12: 3553-35555

Antal A, Kincses TZ, Nitsche MA, Paulus W (2003): Manipulation of phosphene thresholds by transcranial direct current stimulation in man.

Exp Brain Res; 150: 375-378

Antal A, Varga ET, Kincses TZ, Nitsche MA, Paulus W (2004a): Oscillatory brain activity and transcranial direct current stimulation in humans.

Neuroreport; 15: 1307-1310

Antal A, Nitsche MA, Kruse W, Kinczes TZ, Hoffmann KP, Paulus W (2004b): Direct current stimulation over $\mathrm{V} 5$ enhances visuo-motor coordination by improving motion perception in humans. J Cogn Neurosci; 16: 521-527

Antal A, Nitsche MA, Kinczes TZ, Kruse W, Hoffmann KP, Paulus W (2004c): Facilitation of visuo-motor learning by transcranial direct current stimulation of the motor and extrastriate visual areas in humans. Eur J Neurosci; 19: 2888-2892

Antal A, Kriener N, Lang N, Boros K, Paulus W (2011): Cathodal transcranial direct current stimulation of the visual cortex in the prophylactic treatment of migraine.

Cephalalgia; 31: 820-828

Beck H, Goussakov IV, Lie A, Helmstaedter C, Elger CE (2000): Synaptic plasticity in the human dentate gyrus. J Neurosci; 20: 7080-7086 
Bengtson CP, Freitag HE, Weislogel JM, Bading H (2010): Nuclear calcium sensors reveal that repetition of trains of synaptic stimuli boosts nuclear calcium signaling in CA1 pyramidal neurons. Biophys J; 9 9: 4066-4077

Bienenstock EL, Cooper LN, Munro PW (1982): Theory fort the development of neuron selectivity: orientation specificity and binocular interaction in visual cortex. J Neurosci; 2 (1): 32-48

Bindman LJ, Lippold OCJ, Redfearn JWT (1964): The action of brief polarizing currents on the cerebral cortex of the rat (1) during current flow and (2) in the production of long-lasting after-effects. J Physiol; 172: 369-382

Bliss TV, Lomo T (1973): Long-lasting potentiation of synaptic transmission in the dentate area of the anaesthetized rabbit following stimulation of the perforant path.

J Physiol; 232 (2): 331-56

Bliss TV, Collingridge GL (1993): A synaptic model of memory: long-term potentiation in the hippocampus. Nature; $\underline{361}:$ 31-39

Bliss TV, Collingridge GL, Morris RG (2003): Introduction. Long-term potentiation and structure of the issue. Philos Trans R Soc Lond B Biol Sci; $\underline{358}$ : 607-611

Boggio PS, Khoury LP, Martins DC, Martins OE, de Macedo EC, Fregni F (2009): Temporal cortex direct current stimulation enhances performance on visual recognition memory task in Alzheimer disease.

J Neurol Neurosurg Psychiatry; 80 (4): 444-7

Boros K, Poreisz C, Münchau A, Paulus W, Nitsche MA (2008): Premotor transcranial direct current stimulation (tDCS) affects primary motor excitability in humans. Eur J Neurosci; 27: 1292-1300

Callan DE, Schweighofer N (2010): Neural correlates of the spacing effect in explicit verbal semantic encoding support the deficient-processing theory.

Hum Brain Mapp; 4: 645-59. 
Carney MW (1969): Negative polarisation of the brain in the treatment of manic states. Ir J Med Sci; $\underline{8}$ (3): 133-5

Castro-Alamancos MA, Donoghue JP, Connors BW (1995): Different forms of synaptic plasticity in somatosensory and motor areas of the neocortex.

J Neurosci; 15: 5324-5333

Collingridge GL, Kehl SJ, McLennan H (1983): Excitatory amino acids in synaptic transmission in the Schaffer collateral-commissural pathway of the rat hippocampus. J Physiol; 334: 33-46

Cooke SF, Bliss TV (2006): Plasticity in the human central nervous system. Brain; 129: 1659-73

Costain R, Redfearn JC, Lippold OC (1964): A controlled trial of the therapeutic effect of polarization of the brain in depressive illness. Brain J Psychiatry; 110: 786-99

Costa-Mattioli M, Sossin WS, Klann E, Sonnenberg N (2009): Translational control of long-lasting synaptic plasticity and memory. Neuron; $\underline{61}$ (1): 10-26

Creutzfeldt OD, Fromm GH, Kapp H (1962): Influence of transcortical d-c currents on cortical neuronal activity. Exp Neurol; 古: 436-452

Desai NS, Cudmore RH, Nelson SB, and Turrigiano GG (2002): Critical periods for experience-dependent synaptic scaling in the visual cortex.

Nat Neurosci; $\underline{\mathbf{5}}$ : 783-789

Dieckhöfer A, Waberski TD, Nitsche M, Paulus W, Buchner H, Gobbelé R (2006): Transcranial direct current stimulation applied over the somatosensory cortexdifferential effect on low and high frequency SEPs.

Clin Neurophysiol; $\underline{117}$ (10): 2221-2227 
Durand S, Fromy B, Bouyé P, Samuet JL, Abraham P (2002): Vasodilatation in response to repeated anodal current application in the human skin relies on aspirinsensitive mechanisms. J Physiol; 540: 261-269

Feldman DE (2009): Synaptic mechanisms for plasticity in neocortex. Annu Rev Neurosci; 32: 33-55

Flöel A, Rösser N, Michka O, Knecht S, Breitenstein C (2008): Noninvasive brain stimulation improves language learning. J Cogn Neurosci; 20. 1415-1422

Fregni F, Boggio PS, Nitsche M, Bermpohl F, Antal A, Feredoes E, Marcolin MA, Rigonatti SP, Silva MTA, Paulus W, Pascual-Leone A (2005): Anodal transcranial direct current stimulation of prefrontal cortex enhances working memory.

Exp Brain Res; 166: 23-30

Fregni F, Boggio PS, Nitsche MA, Rigonatti SP, Pascual-Leone A (2006a):

Cognitive effects of repeated sessions of transcranial direct current stimulation in patients with depression. Depress Anxiety; 23: 482-484

Fregni F, Boggio PS, Nitsche MA, Marcolin MA, Rigonatti SP, Pascual-Leone A (2006b): Treatment of major depression with transcranial direct current stimulation.

Bipol Disord; $\underline{8}$ : 203-204

Fregni F, Thome-Souza S, Nitsche MA, Freedman SD, Valente KD, Pascual-Leone A (2006c): A controlled clinical trial of cathodal DC polarization in patients with refractory epilepsy. Epilepsia; $\underline{\text { 47: 335-342 }}$

Fregni F, Gimenes R, Valle AC, Ferreira MJL, Rocha RR, Natalle L, Bravo R, Rigonatti SP, Freedmann SD, Nitsche MA, Pascual-Leone A, Boggio PS (2006d): A randomized, sham-controlled. Proof of principle study of transcranial direct current stimulation for the treatment of fibromyalgia. Arthritis \& Rheum; $\underline{\mathbf{4 4}}$ : 3988-3998 
Fregni F, Boggio SP, Santos MC, Lima M, Vieira AL, Rigonatti SP, Silva TA, Barbosa $E R$, Nitsche MA, Pascual-Leone A (2006e): Noninvasive cortical stimulation with transcranial direct current stimulation in Parkinson's Disease.

Mov Disord; 21 (10): 1693-1702

Frey U, Krug M, Reymann KG, Matthies H (1988): Anisomycin, an inhibitor of protein synthesis, blocks late phase of LTP phenomena in the hippocampal CA1 region in vitro. Brain Res; 4 52 (1-2): 57-65

Frey U, Huang YY, Kandel ER (1993): Effects of cAMP simulate a late stage of LTP in hippocampal CA1 neurons. Science; $\underline{260}$ (5114): 1661-1664.

Fricke K, Seeber AA, Thirugnanasambandam N, Paulus W, Rothwell JC (2011):

Time course of the induction of homeostatic plasticity generated by repeated transcranial direct current stimulation of the human motor cortex.

J Neurophysiol; 105 (3): 1141-1149

Gamboa OL, Antal A, Moliadze V, Paulus W (2010): Simply longer is not better: reversal of thetaburst after-effect with prolonged stimulation.

Exp. Brain. Res; 204 (2): 181-187

Gartside IB (1968): Mechanisms of sustained increases of firing rate of neurons in the rat cerebral cortex after polarization: role of protein synthesis.

Nature; 220: 383-384

Gong L, He L, Dong Z, Lu X, Poo M, Zhang X (2011): Postinduction requirement of NMDA receptor activation for late-phase long-term potentiation of developing retinotectal synapses in vivo. J Neurosci; 31 (9): 3328-3335

Hattori Y, Moriwaki A, Hori Y (1990): Biphasic effects of polarizing current on adenosine-sensitive generation of cyclic AMP in rat cerebral cortex.

Neurosci Lett; 116: 320-324

Hebb DO: The organization of behaviour. Wiley, New York 1949 
Hess G, Donoghue JP (1994): Long-term potentiation of horizontal connections provides a mechanism to reorganize cortical motor maps.

J Neurophysiol; 71 (6): 2543-2547

Holmes B, Brogden RN, Heel RC, Speight TM \& Avery GS (1984): Flunarizine. A review of its pharmacodynamic and pharmacokinetic properties and therapeutic use. Drugs; 27: 6-44

Huang YY, Colino A, Selig DK, \& Malenka RC (1992): The influence of prior synaptic activity on the induction of long-term potentiation. Science; 255 : 730-733

Huang YY, Nguyen PV, Abel T, Kandel ER (1996): Long-lasting forms of synaptic potentiation in the mammalian hippocampus. Learn Mem; $\underline{\mathbf{3}}(2-3): 74-85$

Huang YY, Pittenger C, Kandel ER (2004): A form of long-lasting, learning-related synaptic plasticity in the hippocampus induced by heterosynaptic low-frequency pairing. Proc Natl Avad Sci USA; 101 (3): 859-864

Huang YZ, Chen RS, Rothwell JC, Wen HY (2007): The after-effect of human theta burst stimulation is NMDA receptor dependant. Clin Neurophysiol; 118 (5): 10281032

Hummel F, Celnik P, Giraux P, Floel A, Wu WW, Gerloff C, Cohen LG(2005):

Effects of non-invasive cortical stimulation on skilled motor function in chronic stroke. Brain; 128: 490-499

Islam N, Aftabuddin M, Moriwaki A, Hattori Y, Hori Y (1995): Increase in the calcium level following anodal polarization in the rat brain. Brain Res; 684: 206-208

lyer MB, Schleper N, Wassermann EM (2003): Priming stimulation enhances the depressent effect of low-frequency repetitive transcranial magnetic stimulation.

J Neurosci; 23: 10867-10872 
lyer MB, Mattu U, Grafman J, Lomarev M, Sato S, Wassermann EM (2005): Safety and cognitive effect of frontal DC polarization in healthy individuals.

Neurology; $\underline{64}$ (5): 872-875

Kandel ER (2001): The molecular biology of memory storage: a dialogue between genes and synapses. Science; 294 (5544): 1030-1038

Kim M, Huang T, Abel T, Blackwell KT (2010): Temporal sensitivity of protein kinase a activation in late-phase long term potentiation. PLos Comput Biol; $\underline{\mathbf{6}}$ (2): e1000691

Kim SJ, Kim BK, Ko YJ, Bang MS, Kim MH, Han TR (2010): Functional and histologic changes after repeated transcranial direct current stimulation in rat stroke model. J Korean Med Sci; 25 (10): 1499-1505

Kirkwood A, Rioult MC, Bear MF (1996): Experience-dependent modifications of synaptic plasticity in visual cortex. Nature; $\underline{\mathbf{3 8 1}}$ : 526-528

Kornell N, Castel AD, Eich TS, Bjork RA (2010): Spacing as the friend of memory and induction in young and older adults. Psychol Aging; $\underline{25}$ (2): 498-503

Krug M, Matthies R, Wagner M, Brödemann R (1993): Non-opioid antitussives and methadone differentially influence hippocampal long-term potentiation in freely moving rats. Eur J Pharmacol; 231 (3): 355-361

Lang N, Siebner HR, Ernst D, Nitsche MA, Paulus W, Lemon RN, Rothwell JC (2004): Preconditioning with transcranial direct current stimulation sensitizes the motor cortex to rapid-rate transcranial magnetic stimulation and controls the direction

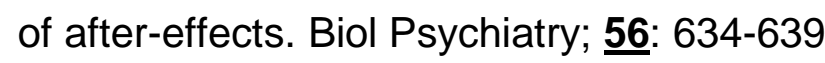

Liebetanz D, Nitsche MA, Tergau F, Paulus W (2002): Pharmacological approach to the mechanisms of transcranial DC-stimulation induced after-effects of human motor cortex excitability. Brain; 125: 2238-2247 
Liebetanz D, Klinker F, Hering D, Koch R, Nitsche MA, Potschka H, Löscher W, Paulus W, Tergau $F$ (2006): Anticonvulsant effects of transcranial direct-current stimulation (tDCS) in the rat cortical ramp model of focal epilepsy.

Epilepsia; 47: 1216-1224

Liebetanz D, Koch R, Mayenfels S, König F, Paulus W, Nitsche MA (2009): Safety limits of cathodal transcranial direct current stimulation in rats.

Clin Neurophysiol; 120: 1161-1167

Lippold OJC, Redfearn JWT (1964): Mental changes resulting from the passage of small direct currents through the human brain. Br J Psychiatry; 110: 768-772

Lolas F (1977): Brain polarization: behavorial and therapeutic effects.

Biol Psychiatry; 12: 37-47

Louis P, Spierings EL (1982): Comparision of flunarizine (Sibelium) and pizotifen (Sandomigran) in migraine treatment: a double-blind study.

Cephalalgia; 2: 197-203



Malenka RC, Bear MF (2004): LTP and LTD: an embarrassment of riches. Neuron; $\underline{44}$ (1):5-21

Malinow R, Malenka RC (2002): AMPA receptor trafficking and synaptic plasticity. Annu Rev Neurosci; 25: 103-126

Martin SJ, Grimwood PD, Morris RG (2000): Synaptic plasticity and memory: an evaluation of the hypothesis. Annu Rev Neurosci; 23: 649-711

Miranda PC, Lomarev M, Hallett M (2006): Modeling the current distribution during transcranial direct current stimulation. Clin Neurophysiol; 117: 1623-1629 
Misonou H, Mohapatra DP, Park EW, Leung V, Zhen D, Misonou K, Anderson AE, Trimmer JS (2004): Regulation of ion channel localization and phosphorylation by neuronal activity. Nat Neurosci; $\underline{7}$ : 711-718

Misonou H, Thompson SM, Cai X (2008): Dynamic Regulation of the Kv2.1 VoltageGated Potassium Channel during Brain Ischemia through Neuroglial Interaction. J Neurosc; 28 (34): 8529-8538

Monte-Silva K, Kuo MF, Liebetanz D, Paulus W, Nitsche MA (2010): Shaping the optimal repetition interval for cathodal transcranial direct current stimulation (tDCS) J Neurophysiol; 103: 1735-1740

Müller JF, Orekhov Y, Liu Y, Ziemann U (2007): Homeostatic plasticity in human motor cortex demonstrated by two consecutive sessions of paired associative stimulation. Eur J Neurosci; $\underline{25}$ (11): 3461-3468

Neves G, Cooke SF, Bliss TV (2008): Synaptic plasticity, memory and the hippocampus: a neural network approach to causality. Nat Rev Neurosci; $\underline{9}$ : 65-75

Nitsche MA, Paulus W (2000): Excitability changes induced in human motor cortex by weak transcranial direct current stimulation. J Physiol; $\underline{\mathbf{5 2 7}}$ : 633-639

Nitsche MA, Paulus W (2001): Sustained excitability elevations induced by transcranial DC motor cortex stimulation in humans. Neurology; $\underline{\mathbf{5 7}}$ : 1899-1901

Nitsche MA, Paulus W (2009): Noninvasive brain stimulation protocols in the treatment of epilepsy: current state and perspectives. Neurotherapeutics; $\underline{\mathbf{6}}$ : 244-250

Nitsche MA, Liebetanz D, Tergau F, Paulus W (2002): Modulation kortikaler Erregbarkeit durch transkranielle Gleichstromstimulation.

Der Nervenarzt; $\underline{73}$ (4): 332-335 
Nitsche MA, Fricke K, Henschke U, Schlitterlau A, Liebetanz D, Lang N, Henning S, Tergau F, Paulus W (2003a): Pharmacological modulation of cortical excitability shifts induced by transcranial DC stimulation. J Physiol; $\underline{553}$ : 293-301

Nitsche MA, Nitsche MS, Klein CC, Tergau F, Rothwell J, Paulus W (2003b):

Level of action of cathodal DC polarisation induced inhibition of the human motor cortex. Clin Neurophys; 114: 600-604

Nitsche MA, Liebetanz D, Antal A, Lang N, Tergau F, Paulus W (2003c): Modulation of cortical excitability by weak direct current stimulation - technical, safety and functional aspects. Suppl Clin Neurophysiol; $\underline{\mathbf{6}}$ : 255-276

Nitsche MA, Schauenburg A, Lang N, Liebetanz D, Exner C, Paulus W, Tergau F (2003d): Facilitation of implicit motor learning by weak transcranial direct current stimulation of the primary motor cortex in the human. J Cog Neurosci; 15: 619-626

Nitsche MA, Jassi W, Liebetanz D, Lang N, Tergau F, Paulus W (2004a): Consolidation of externally induced human motor cortical neuroplasticity by $d$ cycloserine. Neuropsychopharmacology; 29 : 1573-1578

Nitsche MA, Niehaus L, Hoffmann KT, Hengst S, Liebetanz D, Paulus W, Meyer BU (2004b): MRI study of human brain exposed to weak direct current stimulation of the frontal cortex. Clin Neurophys; 115: 2419-2423

Nitsche MA, Seeber A, Frommann K, Klein CC, Nitsche MS, Rochford C, Liebetanz D, Lang N, Antal A, Paulus W, Tergau F (2005): Modulating parameters of excitability during and after transcranial direct current stimulation of the human motor cortex.

J Physiol; 568: 291-303

Nitsche MA, Roth A, Kuo M-F, Fischer AK, Liebetanz D, Lang N, Tergau F, Paulus W (2007): Timing-dependent modulation of associative plasticity by general network excitability in the human motor cortex. J Neurosci; 27: 3807-3812 
Nitsche MA, Cohen LG, Wassermann EM, Priori A, Lang N, Antal A, Paulus W, Hummel F, Boggio PS, Fregni F, Pascual-Leone A (2008): Transcranial direct current stimulation: State of the art 2008. Brain Stimul; 1: 206-223

Nitsche MA, Boggio PS, Fregni F, Pascual-Leone A (2009): Treatment of depression with transcranial direct current stimulation (tDCS): a review. Exp Neurol; 219: 14-19

Nosten-Bertrand M, Errington ML, Murphy KP, Tokugawa Y, Barboni E, Kozlova E, Michalovich D, Morris RG, Silver J, Stewart CL, Bliss TV, Morris RJ (1996): Normal spatial learning despite regional inhibition of LTP in mice lacking Thy-1.

Nature; 379: 826-829

Ohn SH, Park C, Yoo WK, Ko MH, Choi KP, Kim GM, Lee YT, Kim YH (2008):

Time-dependent effect of transcranial direct current stimulation on the enhancement of working memory. Neuroreport; 19 (1): 43-47

Philips GT, Carew TJ (2009): It's all about timing. Cell; $\underline{139}$ (1): 23-25

Poreisz C, Boros K, Antal A, Paulus W (2007): Safety aspects of transcranial direct current stimulation concerning healthy subjects and patients.

Brain Res Bull; $\underline{72}$ (4-6): 208-214

Pozo K, Goda Y (2010): Unraveling mechanisms of homeostatic synaptic plasticity. Neuron; $\underline{66}$ (3): 337-351.

Purpura DP, Mc Murtry JG (1965): Intracellular activities and evoked potential changes during polarization of motor cortex. J Neurophysiol; 28 : 166-185

Reis J, Schambra HM, Cohen LG, Buch ER, Fritsch B, Zarahn E, Celnik PA, Krakauer JW (2009): Noninvasive cortical stimulation enhances motor skill acquisition over multiple days through an effect on consolidation.

Proc Natl Acad Sci USA; 106 (5): 1590-1595 
Reymann KG, Frey JU (2007): The late maintenance of hippocampal LTP: requirements, phases, "synaptic tagging", "late- associativity" and implications. Neuropharmacology; $\underline{\mathbf{5 2}}$ (1): 24-40

Rigonatti SP, Boggio PS, Myczkowski ML, Otta E, Fiquer JT, Ribeiro RB, Nitsche MA, Pascual-Leone A, Fregni $F$ (2008): Transcranial direct current stimulation and fluoxetine for the treatment of depression. Eur Psychiatry; $\underline{\mathbf{2 3}}$ (1): 74-76

Rioult-Pedotti MS, Friedmann D, Hess G, Donoghue JP (1998): Strengthening of horizontal cortical connections following skill learning. Nat Neurosci; 1 (3): 230-234

Rioult-Pedotti MS, Friedman D, Donoghue JP (2000): Learning-induced LTP in neocortex. Science; $\underline{290}$ (5491): 533-536

Rogalewski A, Breitenstein C, Nitsche MA, Paulus W, Knecht S (2004): Transcranial direct current stimulation disrupts tactile perception. Eur J Neurosci; $\underline{20}$ (1): 313-316

Rothwell JC (1993): Evoked potentials, magnetic stimulation studies, and eventrelated potentials. Curr Opin Neurol; $\underline{\mathbf{6}}$ : 715-723

Rush S, Driscoll DA (1968): Current distribution in the brain from surface electrodes. Anaest Analg Curr Res; 47: 717-723

Siebner HR, Ziemann U: Das TMS- Buch, Springer Medizin Verlag, Heidelberg 2007

Siebner HR, Lang N, Rizzo V, Nitsche MA, Paulus W, Lemon RN, Rothwell JC. (2004): Preconditioning of low-frequency repetitive transcranial magnetic stimulation with transcranial direct current stimulation: evidence for homeostatic plasticity in the human motor cortex. J Neurosci; 24: 3379-3385

Sossin WS (2008): Molecular memory traces. Prog Brain Res; $\underline{169:}$ 3-25

Stagg CJ, Nitsche MA (2011): Physiological basis of transcranial direct current Stimulation. The Neuroscientist; 1 (I) $($ ) 37-53 
Stefan K, Kuensch E, Benecke R, Cohen LG, Classen J (2000): Induction of plasticity in the human motor cortex by paired associative stimulation. Brain; 123: 572-584

Stefan K, Kunesch E, Benecke R, Cohen LG, Classen J (2002): Mechanisms of enhancement of human motor cortex excitability induced by interventional paired associative stimulation. J Physiol; $\underline{\mathbf{5 4 3}}$ (Pt 2): 699-708

Steinhoff BJ, Tumani H, Otto M, Mursch K, Wiltfang J, Herrendorf G, Bittermann HJ, Felgenhauer K, Paulus W, Markakis E (1999): Cisternal S 100 protein and neuronspecific Enolase are elevated and site-specific markers in intractable temporal lobe epilepsy. Epilepsy Res; $\underline{\mathbf{3 6}}$ : 75-82

Stoica E \& Enulescu O (1993): The influence of amitryptiline and flunarizine on catecholamine response to light in patients with migraine.

Rom J Neurol Psychiatry; 31: 11-19

Trepel C, Racine R. (1998): Long-term potentiation in the neocortex of the adult, freely moving rat. Cereb Cortex; $\underline{\boldsymbol{8}}$ (8): 719-729

Turrigiano GG (2008): The self-tuning neuron: synaptic scaling of excitatory synapses. Cell; 135 (3): $422-435$

Urban NN, Henze DA, Lewis DA, Barrionuevo G (1996): Properties of LTP induction in the CA3 region of the primate hippocampus. Learn Mem; $\underline{\mathbf{3}}: 86-95$

Vanneste S, Plazier M, Ost J, van der Loo E, Van de Heyning P, De Ridder D (2010):

Bilateral dorsolateral prefrontal cortex modulation for tinnitus by transcranial direct current stimulation: a preliminary clinical study. Exp Brain Res; 202: 779-785

Xue G, Mei L, Chen C, Lu ZL, Poldrack R, Dong Q (2011): Spaced learning enhances subsequent recognition memory by reducing neural repetition suppression. J Cogn Neurosci; $\underline{23}$ (7): 1624-1633 
Zhao C, Dreosti E, Lagnado L (2011): Homeostatic synaptic plasticity through changes in presynaptic calcium influx. J Neurosci; $\underline{31}$ (20): 7492-7496

Ziemann U, Siebner HR (2008): Modifying motor learning through gating and homeostatic metaplasticity. Brain Stimul 2008; 1 (1): 60-66

Ziemann U, llic TV, Pauli C, Meintzschel F, Ruge D (2004): Learning modifies subsequent induction of long-term potentiation-like and long-term depression-like plasticity in human motor cortex. J Neurosci; 24 (7): 1666-1672

Ziemann U, Paulus W, Nitsche MA, Pascuel- Leone a, Byblow WD, Berardelli A, Siebner HR, Classen J, Cohen LG, Rothwell JC (2008): Consensus: Motor cortex plasticity protocols. Brain Stimul; 1 (3): 164-182 


\section{Danksagung}

Ganz besonderer Dank gilt meinem Doktorvater Prof. Dr. med. M. Nitsche für die hervorragende Betreuung der Doktorarbeit, während der er jederzeit ein offenes Ohr für meine Fragen hatte, sowie für die konstruktiven Ratschläge und die schnellen Korrekturen während des Niederschreibens der Dissertation.

Meinen Eltern und meinem Freund möchte ich ganz herzlich für ihre kontinuierliche Unterstützung während des Erstellens der Doktorarbeit danken. Ihre Fürsorge, Ratschläge und Hilfe beim Korrigieren ermöglichten mir erst das Fertigstellen dieser Arbeit.

Außerdem möchte ich Frau Dr. Katía Monte-Silva für die Einarbeitung in die theoretischen Grundlagen und die praktische Anwendung der transkraniellen Gleichstromstimulation, sowie für die Unterstützung bei der Datenauswertung danken.

Ein „Dankeschön“ gilt auch allen Probandinnen und Probanden.

Auch Herrn Prof. Dr. med. W. Paulus als Leiter der Abteilung der Klinischen Neurophysiologie gilt mein Dank. 


\section{Lebenslauf}

Am 12.02.1986 wurde ich in Kassel geboren. Während meiner Schulausbildung besuchte ich die Grundschule in Niederkaufungen und das Friedrichsgymnasium in Kassel. Im Juli 2005 schloss ich meine schulische Ausbildung mit dem Abitur ab.

Im Oktober 2005 begann ich mein Medizinstudium an der Georg-August-Universität Göttingen und bestand den ersten Abschnitt der Ärztlichen Prüfung im September 2007. Im November 2008 nahm ich meine Arbeit an dieser Dissertation in der Abteilung der Klinischen Neurophysiologie in der Neurologischen Medizin auf.

Während des Studiums famulierte ich in der Anästhesie und der Inneren Medizin im Elisabethkrankenhaus in Kassel, in der HNO im Vajira Hospital in Bangkok, in der Psychiatrie am Universitätsklinikum Göttingen und in einer allgemeinmedizinischen Praxis in Kaufungen.

Im August 2010 trat ich in mein Praktisches Jahr ein, welches ich im Psychiatrischen Landeskrankenhaus Lüneburg im Fach Psychiatrie, im St. Martini Krankenhaus Duderstadt im Fach Chirurgie und im Kantonsspital Luzern im Fach Innere Medizin absolvierte.

Mein Studium habe ich mit dem Ablegen des zweiten Abschnitts der Ärztlichen Prüfung am 14. Juni 2012 beendet. 